\title{
WEAK SOLUTIONS AND REGULARITY OF THE INTERFACE IN AN INHOMOGENEOUS FREE BOUNDARY PROBLEM FOR THE $p(x)$-LAPLACIAN
}

\author{
CLAUDIA LEDERMAN AND NOEMI WOLANSKI
}

\begin{abstract}
In this paper we study a one phase free boundary problem for the $p(x)$-Laplacian with non-zero right hand side. We prove that the free boundary of a weak solution is a $C^{1, \alpha}$ surface in a neighborhood of every "flat" free boundary point. We also obtain further regularity results on the free boundary, under further regularity assumptions on the data. We apply these results to limit functions of an inhomogeneous singular perturbation problem for the $p(x)$-Laplacian that we studied in [25].
\end{abstract}

\section{INTRODUCTION}

In this paper we study the following inhomogeneous free boundary problem for the $p(x)$-Laplacian: $u \geq 0$ and

$$
\left(P\left(f, p, \lambda^{*}\right)\right)
$$

$$
\begin{cases}\Delta_{p(x)} u:=\operatorname{div}\left(|\nabla u(x)|^{p(x)-2} \nabla u\right)=f & \text { in }\{u>0\} \\ u=0,|\nabla u|=\lambda^{*}(x) & \text { on } \partial\{u>0\} .\end{cases}
$$

The $p(x)$-Laplacian serves as a model for a stationary non-newtonian fluid with properties depending on the point in the region where it moves. For example, such a situation corresponds to an electrorheological fluid. These are fluids such that their properties depend on the magnitude of the electric field applied to it. In some cases, fluid and Maxwell's equations become uncoupled and a single equation for the $p(x)$-Laplacian appears (see [33]).

The free boundary problem $P\left(f, p, \lambda^{*}\right)$ appears, for instance, in the limit of a singular perturbation problem that may model high activation energy deflagration flames in a fluid with electromagnetic sensitivity (see [25]). When $p(x) \equiv 2$ (in which case the $p(x)$-Laplacian coincides with the Laplacian) this singular perturbation problem was introduced by Zeldovich and Frank-Kamenetski in order to model these kind of flames in [37]. In this latter case, the right hand side $f$ may come from nonlocal effects as well as from external sources (see [23]).

The free boundary problem considered in this paper also appears in an inhomogeneous minimization problem that we study in [26] where we prove that minimizers are weak solutions to $P\left(f, p, \lambda^{*}\right)$.

In the present article we prove that the free boundary $\partial\{u>0\}$ - with $u$ a weak solution of $P\left(f, p, \lambda^{*}\right)$ - is a smooth hypersurface in a neighborhood of every "flat" free boundary point.

Key words and phrases. Free boundary problem, variable exponent spaces, regularity of the free boundary, singular perturbation, inhomogeneous problem.

2010 Mathematics Subject Classification. 35R35, 35B65, 35J60, 35J70.

Supported by the Argentine Council of Research CONICET under the project PIP625, Res. 960/12, UBACYT 20020100100496 and ANPCyT PICT 2012-0153. 
The notion of weak solution used in this paper is such that it also includes the limits of the singular perturbation problem described above, that we studied in [25], under suitable nondegeneracy conditions.

More precisely, in the present work we prove that the free boundary of a weak solution to $P\left(f, p, \lambda^{*}\right)$ (see Definition 2.2) is a $C^{1, \alpha}$ surface near flat free boundary points (Theorems 4.1, 4.2 and 4.3). As a consequence we get that the free boundary is $C^{1, \alpha}$ in a neighborhood of every point in the reduced free boundary (Theorem 4.4). We also obtain further regularity results on the free boundary, under further regularity assumptions on the data (Corollary 4.1).

In the particular situation of the minimization problem mentioned above, we prove in [26] that the set of singular free boundary points has null $\mathcal{H}^{N-1}$-measure.

The basic ideas we follow in this paper to prove the regularity of the free boundary of a weak solution were introduced by Alt and Caffarelli in the seminal paper [1], where the case of distributional weak solutions of $P\left(f, p, \lambda^{*}\right)$ with $p(x) \equiv 2$ and $f \equiv 0$ was studied. The treatment of a quasilinear equation was first done in [2] for the uniformly elliptic case. Then, the $p$-Laplacian $(p(x) \equiv p)$ was treated in [8]. The main difference being that a control of $|\nabla u|$ from below close to the free boundary is needed in order to be able to work with linear equations with the ideas of [2]. Both [2] and [8] deal with minimizers that are weak solutions in the stronger sense of [1]. A notion of weak solution similar to the one in the present paper was first considered in [29]. The case of a variable power $p(x)$ was considered in [16] still for minimizers and in the homogeneous case $f \equiv 0$. The linear inhomogeneous case was treated in [18] and [21] for minimizers.

We point out that the regularity of the free boundary for the inhomogeneous problem $f \not \equiv 0$ had not been obtained even in the case of $p(x) \equiv p$.

For other references related to the free boundary problem under consideration in this paper we would like to refer the reader to [3], [4], [5], [9], [10], [11], [27], [28], [30], [31], [32], [34], [35] and the references therein. This list is by no means exhaustive.

An outline of the paper is as follows: in Section 2 we define the notion of weak solution to the free boundary problem $P\left(f, p, \lambda^{*}\right)$ and we derive some properties of weak solutions. In Section 3 we study the behavior of weak solutions to the free boundary problem $P\left(f, p, \lambda^{*}\right)$ near "flat" free boundary points. In Section 4 we study the regularity of the free boundary for weak solutions to the free boundary problem $P\left(f, p, \lambda^{*}\right)$. In Section 5 we present an application of these results to limit functions of the singular perturbation problem that we studied in [25]. Our results apply to limit functions satisfying suitable conditions that are fulfilled, for instance, under the situation we considered in $[26]$.

1.1. Preliminaries on Lebesgue and Sobolev spaces with variable exponent. Let $p: \Omega \rightarrow$ $[1, \infty)$ be a measurable bounded function, called a variable exponent on $\Omega$ and denote $p_{\max }=$ $\operatorname{esssup} p(x)$ and $p_{\min }=\operatorname{essinf} p(x)$. We define the variable exponent Lebesgue space $L^{p(\cdot)}(\Omega)$ to consist of all measurable functions $u: \Omega \rightarrow \mathbb{R}$ for which the modular $\varrho_{p(\cdot)}(u)=\int_{\Omega}|u(x)|^{p(x)} d x$ is finite. We define the Luxemburg norm on this space by

$$
\|u\|_{L^{p(\cdot)}(\Omega)}=\|u\|_{p(\cdot)}=\inf \left\{\lambda>0: \varrho_{p(\cdot)}(u / \lambda) \leq 1\right\} .
$$

This norm makes $L^{p(\cdot)}(\Omega)$ a Banach space. 
There holds the following relation between $\varrho_{p(\cdot)}(u)$ and $\|u\|_{L^{p(\cdot)}}$ :

$$
\begin{aligned}
\min \left\{\left(\int_{\Omega}|u|^{p(x)} d x\right)^{1 / p_{\min }}\right. & \left.,\left(\int_{\Omega}|u|^{p(x)} d x\right)^{1 / p_{\max }}\right\} \leq\|u\|_{L^{p(\cdot)}(\Omega)} \\
& \leq \max \left\{\left(\int_{\Omega}|u|^{p(x)} d x\right)^{1 / p_{\min }},\left(\int_{\Omega}|u|^{p(x)} d x\right)^{1 / p_{\max }}\right\} .
\end{aligned}
$$

Moreover, the dual of $L^{p(\cdot)}(\Omega)$ is $L^{p^{\prime}(\cdot)}(\Omega)$ with $\frac{1}{p(x)}+\frac{1}{p^{\prime}(x)}=1$.

Let $W^{1, p(\cdot)}(\Omega)$ denote the space of measurable functions $u$ such that $u$ and the distributional derivative $\nabla u$ are in $L^{p(\cdot)}(\Omega)$. The norm

makes $W^{1, p(\cdot)}$ a Banach space.

$$
\|u\|_{1, p(\cdot)}:=\|u\|_{p(\cdot)}+\||\nabla u|\|_{p(\cdot)}
$$

The space $W_{0}^{1, p(\cdot)}(\Omega)$ is defined as the closure of the $C_{0}^{\infty}(\Omega)$ in $W^{1, p(\cdot)}(\Omega)$.

For more about these spaces, see $[12,20]$ and the references therein.

1.2. Preliminaries on solutions to $p(x)$-Laplacian. Let $p(x)$ be as above and let $g \in L^{\infty}(\Omega)$. We say that $u$ is a solution to

$$
\Delta_{p(x)} u=g(x) \text { in } \Omega
$$

if $u \in W^{1, p(\cdot)}(\Omega)$ and, for every $\varphi \in W_{0}^{1, p(\cdot)}(\Omega)$, there holds that

$$
\int_{\Omega}|\nabla u(x)|^{p(x)-2} \nabla u \cdot \nabla \varphi d x=-\int_{\Omega} \varphi g(x) d x .
$$

Under the assumptions of the present paper (see 1.3 below) it follows from [36] that $u \in L_{\text {loc }}^{\infty}(\Omega)$.

For any $x \in \Omega, \xi, \eta \in \mathbb{R}^{N}$ fixed we have the following inequalities

$$
\begin{array}{ll}
|\eta-\xi|^{p(x)} \leq C\left(|\eta|^{p(x)-2} \eta-|\xi|^{p(x)-2} \xi\right)(\eta-\xi) & \text { if } p(x) \geq 2, \\
|\eta-\xi|^{2}(|\eta|+|\xi|)^{p(x)-2} \leq C\left(|\eta|^{p(x)-2} \eta-|\xi|^{p(x)-2} \xi\right)(\eta-\xi) & \text { if } p(x)<2 .
\end{array}
$$

These inequalities imply that the function $A(x, \xi)=|\xi|^{p(x)-2} \xi$ is strictly monotone. Then, the comparison principle for the $p(x)$-Laplacian holds since it follows from the monotonicity of $A(x, \xi)$.

1.3. Assumptions. Throughout the paper we let $\Omega \subset \mathbb{R}^{N}$ be a domain.

Assumptions on $p(x)$. We assume that the function $p(x)$ verifies

$$
1<p_{\min } \leq p(x) \leq p_{\max }<\infty, \quad x \in \Omega .
$$

Unless otherwise stated, we assume that $p(x)$ is Lipschitz continuous in $\Omega$. In some results we assume further that $p \in W^{1, \infty}(\Omega) \cap W^{2, q}(\Omega)$.

Assumptions on $\lambda^{*}(x)$. We assume that the function $\lambda^{*}$ is continuous in $\Omega$ and verifies

$$
0<\lambda_{\min } \leq \lambda^{*}(x) \leq \lambda_{\max }<\infty, \quad x \in \Omega .
$$

In our main results $\lambda^{*}(x)$ is Hölder continuous in $\Omega$.

Assumptions on $f(x)$. We assume that $f \in L^{\infty}(\Omega)$. In some results we assume further that $f \in W^{1, q}(\Omega)$. 


\subsection{Notation.}

- $N$ spatial dimension

- $\Omega \cap \partial\{u>0\} \quad$ free boundary

- $|S| \quad N$-dimensional Lebesgue measure of the set $S$

- $\mathcal{H}^{N-1} \quad(N-1)$-dimensional Hausdorff measure

- $B_{r}\left(x_{0}\right) \quad$ open ball of radius $r$ and center $x_{0}$

- $B_{r} \quad$ open ball of radius $r$ and center 0

- $B_{r}^{+}=B_{r} \cap\left\{x_{N}>0\right\}, \quad B_{r}^{-}=B_{r} \cap\left\{x_{N}<0\right\}$

- $B_{r}^{\prime}\left(x_{0}\right) \quad$ open ball of radius $r$ and center $x_{0}$ in $\mathbb{R}^{N-1}$

- $B_{r}^{\prime} \quad$ open ball of radius $r$ and center 0 in $\mathbb{R}^{N-1}$

- $f_{B_{r}\left(x_{0}\right)} u=\frac{1}{\left|B_{r}\left(x_{0}\right)\right|} \int_{B_{r}\left(x_{0}\right)} u d x$

- $f_{\partial B_{r}\left(x_{0}\right)} u=\frac{1}{\mathcal{H}^{N-1}\left(\partial B_{r}\left(x_{0}\right)\right)} \int_{\partial B_{r}\left(x_{0}\right)} u d \mathcal{H}^{N-1}$

- $\chi_{S} \quad$ characteristic function of the set $S$

- $u^{+}=\max (u, 0), u^{-}=\max (-u, 0)$

- $\langle\xi, \eta\rangle$ and $\xi \cdot \eta \quad$ both denote scalar product in $\mathbb{R}^{N}$

\section{Weak solutions to the free boundary problem $P\left(f, p, \lambda^{*}\right)$}

In this section we define the notion of weak solution to the free boundary problem $P\left(f, p, \lambda^{*}\right)$.

We also derive some properties of the weak solutions to problem $P\left(f, p, \lambda^{*}\right)$, which will be used in the next sections, where a theory for the regularity of the free boundary for weak solutions will be developed.

In all the results of this section $p(x)$ will be a Lipschitz continuous function.

We first need

Definition 2.1. Let $u$ be a continuous and nonnegative function in a domain $\Omega \subset \mathbb{R}^{N}$. We say that $\nu$ is the exterior unit normal to the free boundary $\Omega \cap \partial\{u>0\}$ at a point $x_{0} \in \Omega \cap \partial\{u>0\}$ in the measure theoretic sense, if $\nu \in \mathbb{R}^{N},|\nu|=1$ and

$$
\lim _{r \rightarrow 0} \frac{1}{r^{N}} \int_{B_{r}\left(x_{0}\right)}\left|\chi_{\{u>0\}}-\chi_{\left\{x /\left\langle x-x_{0}, \nu\right\rangle<0\right\}}\right| d x=0 .
$$

Then we have

Definition 2.2. Let $\Omega \subset \mathbb{R}^{N}$ be a domain. Let $p$ be a measurable function in $\Omega$ with $1<p_{\min } \leq$ $p(x) \leq p_{\max }<\infty, \lambda^{*}$ continuous in $\Omega$ with $0<\lambda_{\min } \leq \lambda^{*}(x) \leq \lambda_{\max }<\infty$ and $f \in L^{\infty}(\Omega)$. We call $u$ a weak solution of $P\left(f, p, \lambda^{*}\right)$ in $\Omega$ if

(1) $u$ is continuous and nonnegative in $\Omega, u \in W^{1, p(\cdot)}(\Omega)$ and $\Delta_{p(x)} u=f$ in $\Omega \cap\{u>0\}$.

(2) For $D \subset \subset \Omega$ there are constants $c_{\min }=c_{\min }(D), C_{\max }=C_{\max }(D), r_{0}=r_{0}(D), 0<c_{\min } \leq$ $C_{\max }, r_{0}>0$, such that for balls $B_{r}(x) \subset D$ with $x \in \partial\{u>0\}$ and $0<r \leq r_{0}$

$$
c_{\min } \leq \frac{1}{r} \sup _{B_{r}(x)} u \leq C_{\max }
$$

(3) For $\mathcal{H}^{N-1}$ a.e. $x_{0} \in \partial_{\text {red }}\{u>0\}$ (this is, for $\mathcal{H}^{N-1}$-almost every point $x_{0} \in \Omega \cap \partial\{u>0\}$ such that $\Omega \cap \partial\{u>0\}$ has an exterior unit normal $\nu\left(x_{0}\right)$ in the measure theoretic sense) $u$ has the asymptotic development

$$
u(x)=\lambda^{*}\left(x_{0}\right)\left\langle x-x_{0}, \nu\left(x_{0}\right)\right\rangle^{-}+o\left(\left|x-x_{0}\right|\right) .
$$


(4) For every $x_{0} \in \Omega \cap \partial\{u>0\}$,

$$
\limsup _{\substack{x \rightarrow x_{0} \\ u(x)>0}}|\nabla u(x)| \leq \lambda^{*}\left(x_{0}\right) .
$$

If there is a ball $B \subset\{u=0\}$ touching $\Omega \cap \partial\{u>0\}$ at $x_{0}$ then,

$$
\limsup _{\substack{x \rightarrow x_{0} \\ u(x)>0}} \frac{u(x)}{\operatorname{dist}(x, B)} \geq \lambda^{*}\left(x_{0}\right) .
$$

Definition 2.3. Let $v$ be a continuous nonnegative function in a domain $\Omega \subset \mathbb{R}^{N}$. We say that $v$ is nondegenerate at a point $x_{0} \in \Omega \cap\{v=0\}$ if there exist $c>0, \bar{r}_{0}>0$ such that one of the following conditions holds:

$$
\begin{gathered}
f_{B_{r}\left(x_{0}\right)} v d x \geq c r \quad \text { for } 0<r \leq \bar{r}_{0}, \\
f_{\partial B_{r}\left(x_{0}\right)} v d x \geq c r \quad \text { for } 0<r \leq \bar{r}_{0}, \\
\sup _{B_{r}\left(x_{0}\right)} v \geq c r \quad \text { for } 0<r \leq \bar{r}_{0} .
\end{gathered}
$$

We say that $v$ is uniformly nondegenerate on a set $\Gamma \subset \Omega \cap\{v=0\}$ in the sense of (2.3) (resp. (2.4), (2.5)) if the constants $c$ and $\bar{r}_{0}$ in (2.3) (resp. (2.4), (2.5)) can be taken independent of the point $x_{0} \in \Gamma$.

Remark 2.1. Assume that $v \geq 0$ is locally Lipschitz continuous in a domain $\Omega \subset \mathbb{R}^{N}, v \in$ $W^{1, p(\cdot)}(\Omega)$ with $\Delta_{p(x)} v \geq f \chi_{\{v>0\}}$, where $f \in L^{\infty}(\Omega), 1<p_{\min } \leq p(x) \leq p_{\max }<\infty$ and $p(x)$ is Lipschitz continuous. Then the three concepts of nondegeneracy in Definition 2.3 are equivalent (for the idea of the proof, see Remark 3.1 in [22], where the case $p(x) \equiv 2$ and $f \equiv 0$ is treated).

We will now derive some properties of the weak solutions.

Lemma 2.1. If $u$ satisfies the hypothesis (1) of Definition 2.2 then $\lambda=\lambda_{u}:=\Delta_{p(x)} u-f \chi_{\{u>0\}}$ is a nonnegative Radon measure with support on $\Omega \cap \partial\{u>0\}$.

Proof. The proof follows as in the case $p(x) \equiv 2$, that was done in [24], Lemma 2.1.

Proposition 2.1. Assume that u satisfies hypothesis (1) of Definition 2.2. Assume moreover that $u \in L^{\infty}(\Omega),\|\nabla p\|_{L^{\infty}} \leq L$ and there exist constants $C_{0}>0, \hat{r}_{0}>0$ such that if $x \in \Omega \cap \partial\{u>0\}$, $B_{r}(x) \subset \Omega$ and $r \leq \hat{r}_{0}$, then

$$
\sup _{B_{r(x)}} u \leq C_{0} r .
$$

Then, $u$ is locally Lipschitz. Moreover, for any $D \subset \subset \Omega$ the Lipschitz constant of $u$ in $D$ can be estimated by a constant $C$ depending only on $N, p_{\min }, p_{\max }, L, \operatorname{dist}(D, \partial \Omega),\|u\|_{L^{\infty}(\Omega)},\|f\|_{L^{\infty}(\Omega)}, C_{0}$ and $\hat{r}_{0}$.

Proof. We will find a constant $C$ such that $|\nabla u| \leq C$ in $D \cap\{u>0\}$. Let $r_{1}=\operatorname{dist}(D, \partial \Omega)$ and $y \in D \cap\{u>0\}$ such that $\operatorname{dist}(y, \partial\{u>0\})<\min \left\{\frac{\hat{r}_{0}}{2}, \frac{r_{1}}{3}, 1\right\}$. Let $\bar{x} \in \partial\{u>0\}$ such that $r=\operatorname{dist}(y, \partial\{u>0\})=|\bar{x}-y|$. Then $B_{r}(y) \subset B_{2 r}(\bar{x})$ and thus,

$$
\frac{1}{r} \sup _{B_{r(y)}} u \leq \frac{1}{r} \sup _{B_{2 r(\bar{x})}} u \leq 2 C_{0} .
$$


We will show that there exists $\tilde{C}$ such that

$$
|\nabla u(y)| \leq \tilde{C}\left(1+\left(\frac{1}{r} \sup _{B_{r}(y)} u\right)^{p_{\max } / p_{\min }}\right)
$$

In fact, let $v(z)=\frac{1}{r} u(y+r z)$. Then, $\|v\|_{L^{\infty}\left(B_{1}\right)} \leq 2 C_{0}$ and $\Delta_{\bar{p}(x)} v=\bar{f}$ in $B_{1}$, with $\bar{p}(z)=$ $p(y+r z), \bar{f}(z)=r f(y+r z)$. There holds that $p_{\min } \leq \bar{p}(x) \leq p_{\max },\|\nabla \bar{p}\|_{L^{\infty}} \leq L$ and $\|\bar{f}\|_{L^{\infty}\left(B_{1}\right)} \leq$ $\|f\|_{L^{\infty}(\Omega)}$, if $0<r<1$. By the local results in [14] it follows that $v \in C_{\text {loc }}^{1, \alpha}\left(B_{1}\right)$ and then, there exists $C_{1}>0$ such that $\|\nabla v\|_{C^{\alpha}\left(B_{1 / 2}\right)} \leq C_{1}$. Therefore, if $z \in B_{1 / 2}(0)$

$$
|\nabla v(0)| \leq C_{2}+|\nabla v(z)|
$$

and thus, if $x \in B_{r / 2}(y)$,

$$
|\nabla u(y)| \leq C_{2}+|\nabla u(x)|
$$

If $|\nabla u(y)| \leq 1$, the desired bound follows. If $|\nabla u(y)| \geq 1$, we get

$$
|\nabla u(y)|^{p_{\min }} \leq|\nabla u(y)|^{p(x)} \leq C_{3}\left(1+|\nabla u(x)|^{p(x)}\right) .
$$

Integrating for $x \in B_{r / 2}(y)$, we obtain

$$
|\nabla u(y)|^{p_{\min }} \leq C_{3}\left(1+f_{B_{r / 2}(y)}|\nabla u(x)|^{p(x)}\right) .
$$

Applying Cacciopoli type inequality (see [14], Lemma 3.1, (3.5)) we have, for some constants $C_{4}$ and $R_{0}$ that, if $r \leq R_{0}$ and $\omega=f_{B_{r}(y)} u(x)$,

$$
\begin{aligned}
|\nabla u(y)|^{p_{\min }} & \leq C_{4}\left(1+f_{B_{r}(y)}\left(\frac{|u(x)-\omega|}{r}\right)^{p(x)}\right) \\
& \leq C_{4}\left(2+\left(\frac{2}{r} \sup _{B_{r}(y)} u\right)^{p_{\max }}\right) .
\end{aligned}
$$

This gives the result in case $\operatorname{dist}(y, \partial\{u>0\})<R_{1}$, with $R_{1}=\min \left\{R_{0}, \frac{\hat{r}_{0}}{2}, \frac{r_{1}}{3}, 1\right\}$. If, on the other hand, $\operatorname{dist}(y, \partial\{u>0\}) \geq R_{1}$, the local results of [14] give

$$
|\nabla u(y)| \leq \bar{C},
$$

for a constant $\bar{C}$ depending on $N, p_{\min }, p_{\max }, L,\|u\|_{L^{\infty}(\Omega)},\|f\|_{L^{\infty}(\Omega)}, R_{1}$. We thus obtain the desired estimate.

Lemma 2.2. Assume that $u$ satisfies hypotheses (1) and (2) of Definition 2.2. For $D \subset \subset \Omega$ there are constants $0<\tilde{c}_{\min } \leq \tilde{C}_{\max }$ and $\tilde{r}_{0}>0$ such that for balls $B_{r}(x) \subset D$ with $x \in \partial\{u>0\}$ and $0<r \leq \tilde{r}_{0}$

$$
\tilde{c}_{\min } \leq \frac{1}{r} f_{B_{r}(x)} u d x \leq \tilde{C}_{\max }
$$

Proof. The result follows from Proposition 2.1, Lemma 2.1 and Remark 2.1.

Lemma 2.3. Assume that $u$ satisfies hypotheses (1) and (2) of Definition 2.2.

Then, for any domain $D \subset \subset \Omega$ there exist constants $c$ and $\bar{r}_{0}>0$, with $0<c<1$, depending on $\|\nabla u\|_{L^{\infty}(D)},\|f\|_{L^{\infty}(D)} r_{0}, p_{\min }, p_{\max },\|\nabla p\|_{L^{\infty}(D)}$ and $c_{\min }$, such that for every $B_{r} \subset D$, centered at the free boundary with $0<r \leq \bar{r}_{0}$ we have

$$
\frac{\left|B_{r} \cap\{u>0\}\right|}{\left|B_{r}\right|} \geq c
$$


Proof. We first notice that, by Proposition 2.1 and Lemma 2.2, $u$ is locally Lipschitz and (2.6) holds. Let $B_{r}\left(x_{0}\right) \subset D$ with $x_{0} \in \partial\{u>0\}$. We observe that $u(x) \leq r\|\nabla u\|_{L^{\infty}(D)}$ in $\{u>0\} \cap B_{r}\left(x_{0}\right)$. Therefore, for $0<r \leq \tilde{r}_{0}$

$$
\tilde{c}_{\min } \leq \frac{1}{r} f_{B_{r}\left(x_{0}\right)} u d x \leq\|\nabla u\|_{L^{\infty}(D)} \frac{\left|B_{r}\left(x_{0}\right) \cap\{u>0\}\right|}{\left|B_{r}\left(x_{0}\right)\right|} .
$$

Remark 2.2. Assume that $u$ satisfies hypotheses (1) and (2) of Definition 2.2. It follows from Lemma 2.3 that the free boundary has Lebesgue measure zero.

Lemma 2.4. Assume that u satisfies hypotheses (1) and (2) of Definition 2.2.

Then for any domain $D \subset \subset \Omega$ there exist constants $c, C$ and $\bar{r}_{0}$ depending on $N, p_{\min }, p_{\max }$, $\|\nabla p\|_{L^{\infty}(D)},\|f\|_{L^{\infty}(D)},\|\nabla u\|_{L^{\infty}(D)}, c_{\min }, C_{\max }$ and $r_{0}$ such that, for every $B_{r} \subset D$ centered at the free boundary, with $r \leq \bar{r}_{0}$, we have

$$
c r^{N-1} \leq \int_{B_{r}} d \lambda \leq C r^{N-1}
$$

Here $\lambda=\lambda_{u}$ is as in Lemma 2.1.

Proof. Let $\xi \in C_{0}^{\infty}(\Omega), \xi \geq 0$. Then,

$$
\int_{\Omega} \xi d \lambda=-\int|\nabla u|^{p(x)-2} \nabla u \cdot \nabla \xi d x-\int_{\{u>0\}} f \xi d x .
$$

Approximating $\chi_{B_{r}}$ from below by a sequence $\left\{\xi_{n}\right\}$ in $C_{0}^{\infty}(\Omega)$ such that $0 \leq \xi_{n} \leq 1, \xi_{n}=1$ in $B_{r-\frac{1}{n}}$ and $\left|\nabla \xi_{n}\right| \leq C_{N} n$ and using that $u$ is locally Lipschitz, we have that

$$
\begin{aligned}
-\int|\nabla u|^{p(x)-2} \nabla u \cdot \nabla \xi_{n} d x-\int_{\{u>0\}} f \xi_{n} d x & \leq C_{0} n\left|B_{r} \backslash B_{r-\frac{1}{n}}\right|+\left.|| f\right|_{L^{\infty}(D)}\left|B_{r}\right| \\
& \leq C_{1} r^{N-1},
\end{aligned}
$$

if $r \leq 1$, with $C_{0}=C_{0}\left(p_{\max },\|\nabla u\|_{L^{\infty}(D)}, N\right)$ and $C_{1}=C_{1}\left(p_{\max },\|\nabla u\|_{L^{\infty}(D)}, N,\|f\|_{L^{\infty}(D)}\right)$.

Then, as

$$
\int_{\Omega} \xi_{n} d \lambda \rightarrow \int_{B_{r}} d \lambda
$$

the bound from above holds.

Let us now prove the bound from below. Arguing by contradiction we assume that there exists a sequence of functions $u_{k}$ satisfying hypotheses (1) and (2) of Definition 2.2 with power $p_{k}(x)$ and right hand side $f_{k}(x)$, with $p_{\min } \leq p_{k}(x) \leq p_{\max },\left\|\nabla p_{k}\right\|_{L^{\infty}(D)} \leq L_{1},\left\|f_{k}\right\|_{L^{\infty}(D)} \leq L_{2}$ and $\left\|\nabla u_{k}\right\|_{L^{\infty}(D)} \leq L_{0}$, and balls $B_{r_{k}}\left(x_{k}\right) \subset D$, with $x_{k} \in \partial\left\{u_{k}>0\right\}$ and $r_{k} \rightarrow 0$ with $\lambda_{k}=\Delta_{p_{k}(x)} u_{k}-$ $f_{k} \chi_{\left\{u_{k}>0\right\}}$ satisfying that $\int_{B_{r_{k}}\left(x_{k}\right)} d \lambda_{k} \leq \varepsilon_{k} r_{k}{ }^{N-1}$ with $\varepsilon_{k} \rightarrow 0$. Let $v_{k}(x)=\frac{u_{k}\left(x_{k}+r_{k} x\right)}{r_{k}}$. As the $v_{k}^{\prime} s$ are uniformly Lipschitz in $B_{1}(0)$ and $v_{k}(0)=0$, we can assume that $v_{k} \rightarrow v_{0}$ uniformly in $B_{1 / 2}$. We can also assume that $x_{k} \rightarrow x_{0} \in \bar{D}$.

We have $v_{k} \geq 0$ and $\Delta_{\bar{p}_{k}(x)} v_{k}=\bar{f}_{k}$ in $B_{1}(0) \cap\left\{v_{k}>0\right\}$, with $\bar{p}_{k}(x)=p_{k}\left(x_{k}+r_{k} x\right), \bar{f}_{k}(x)=$ $r_{k} f_{k}\left(x_{k}+r_{k} x\right)$. We can assume that $\bar{p}_{k} \rightarrow p_{0} \in \mathbb{R}$ uniformly on compact subsets of $B_{1}(0)$.

We claim that $\nabla v_{k} \rightarrow \nabla v_{0}$ a.e. in $B_{1 / 2}$. In fact, on one hand, by the interior Hölder gradient estimates, we have that $\nabla v_{k} \rightarrow \nabla v_{0}$ uniformly on compact subsets of $\left\{v_{0}>0\right\}$.

On the other hand, if $B_{r}(\bar{x}) \subset\left\{v_{0} \equiv 0\right\} \cap B_{1 / 2}(0)$, then $B_{r / 2}(\bar{x}) \cap \partial\left\{v_{k}>0\right\}=\emptyset$ for large $k$ by the nondegeneracy. So, either $B_{r / 2}(\bar{x}) \subset\left\{v_{k} \equiv 0\right\}$ for a subsequence, or else $v_{k}>0$ in $B_{r / 2}(\bar{x})$ 
for large $k$. In any case, $\nabla v_{k} \rightarrow \nabla v_{0}$ uniformly in $B_{r / 4}(\bar{x})$. Now observing that, with the same argument used in Remark 2.2, we get that $\left|B_{1 / 2}(0) \cap \partial\left\{v_{0}>0\right\}\right|=0$, the claim follows.

Then, for all $\xi \in C_{0}^{\infty}\left(B_{1 / 2}\right), \xi \geq 0$,

$$
-\int_{B_{1 / 2}}\left|\nabla v_{0}\right|^{p_{0}-2} \nabla v_{0} \cdot \nabla \xi=\lim _{k \rightarrow \infty}\left(-\int_{B_{1 / 2}}\left|\nabla v_{k}\right|^{\bar{p}_{k}(x)-2} \nabla v_{k} \cdot \nabla \xi-\int_{B_{1 / 2}} \bar{f}_{k} \xi \chi_{\left\{v_{k}>0\right\}}\right) .
$$

On the other hand, denoting $\varphi(y)=\xi\left(\frac{y-x_{k}}{r_{k}}\right)$, we have

$$
-\int_{B_{1 / 2}}\left|\nabla v_{k}\right|^{\bar{p}_{k}(x)-2} \nabla v_{k} \cdot \nabla \xi-\int_{B_{1 / 2}} \bar{f}_{k} \xi \chi_{\left\{v_{k}>0\right\}}=\frac{1}{r_{k}^{N-1}} \int_{B_{r_{k} / 2}\left(x_{k}\right)} \varphi d \lambda_{k} \leq\|\varphi\|_{L^{\infty}\left(B_{r_{k} / 2}\left(x_{k}\right)\right)} \varepsilon_{k} \rightarrow 0 .
$$

Therefore $\Delta_{p_{0}} v_{0}=0$ in $B_{1 / 2}$. But $v_{0} \geq 0$ and $v_{0}(0)=0$, so that by the Harnack inequality we have $v_{0}=0$ in $B_{1 / 2}$.

On the other hand, $0 \in \partial\left\{v_{k}>0\right\}$, and by the nondegeneracy, we have

$$
\int_{B_{1 / 4}} v_{k} \geq c>0
$$

Thus,

$$
\int_{B_{1 / 4}} v_{0} \geq c>0
$$

which is a contradiction.

The next result gives a representation formula for weak solutions. We will denote by $\mathcal{H}^{N-1}\lfloor\partial\{u>$ $0\}$ the measure $\mathcal{H}^{N-1}$ restricted to the set $\partial\{u>0\}$.

Theorem 2.1. Assume that u satisfies hypotheses (1) and (2) of Definition 2.2. Then, 1) $\mathcal{H}^{N-1}(D \cap \partial\{u>0\})<\infty$, for every $D \subset \subset \Omega$.

2) There exist a borelian function $q_{u}$ defined on $\Omega \cap \partial\{u>0\}$ such that

$$
\Delta_{p(x)} u-f \chi_{\{u>0\}}=q_{u} \mathcal{H}^{N-1}\lfloor\partial\{u>0\} .
$$

3) For every $D \subset \subset \Omega$ there exist $C>0, c>0$ and $r_{1}>0$ such that

$$
c r^{N-1} \leq \mathcal{H}^{N-1}\left(B_{r}\left(x_{0}\right) \cap \partial\{u>0\}\right) \leq C r^{N-1}
$$

for balls $B_{r}\left(x_{0}\right) \subset D$ with $x_{0} \in D \cap \partial\{u>0\}$ and $0<r<r_{1}$ and, in addition, 4) $c \leq q_{u} \leq C$ in $D \cap \partial\{u>0\}$.

Proof. The result follows as Theorem 4.5 in [1].

Remark 2.3. Assume that $u$ satisfies hypotheses (1) and (2) of Definition 2.2. It follows from Theorem 2.1 that the set $\Omega \cap\{u>0\}$ has finite perimeter locally in $\Omega$ (see [15] 4.5.11). That is, $\mu_{u}:=-\nabla \chi_{\{u>0\}}$ is a Borel measure, and the total variation $\left|\mu_{u}\right|$ is a Radon measure. In this situation, we define the reduced boundary as in [15], 4.5.5. (see also [13]) by, $\partial_{\text {red }}\{u>0\}:=\{x \in$ $\left.\Omega \cap \partial\{u>0\} /\left|\nu_{u}(x)\right|=1\right\}$, where $\nu_{u}(x)$ is the unit vector with

$$
\int_{B_{r}(x)}\left|\chi_{\{u>0\}}-\chi_{\left\{y /\left\langle y-x, \nu_{u}(x)\right\rangle<0\right\}}\right|=o\left(r^{N}\right)
$$

for $r \rightarrow 0$, if such a vector exists, and $\nu_{u}(x)=0$ otherwise. By the results in [15] Theorem 4.5.6, we have

$$
\mu_{u}=\nu_{u} \mathcal{H}^{N-1}\left\lfloor\partial_{\text {red }}\{u>0\} .\right.
$$


We also have the following result on blow up sequences

Lemma 2.5. Assume that $u$ satisfies hypotheses (1) and (2) of Definition 2.2. Let $B_{\rho_{k}}\left(x_{k}\right) \subset \Omega$ be a sequence of balls with $\rho_{k} \rightarrow 0, x_{k} \rightarrow x_{0} \in \Omega$ and $u\left(x_{k}\right)=0$. Let us consider the blow-up sequence with respect to $B_{\rho_{k}}\left(x_{k}\right)$. That is,

$$
u_{k}(x):=\frac{1}{\rho_{k}} u\left(x_{k}+\rho_{k} x\right) .
$$

Then, there exists a blow-up limit $u_{0}: \mathbb{R}^{N} \rightarrow \mathbb{R}$ such that, for a subsequence,

(1) $u_{k} \rightarrow u_{0}$ in $C_{\text {loc }}^{\alpha}\left(\mathbb{R}^{N}\right)$ for every $0<\alpha<1$,

(2) $\partial\left\{u_{k}>0\right\} \rightarrow \partial\left\{u_{0}>0\right\}$ locally in Hausdorff distance,

(3) $\nabla u_{k} \rightarrow \nabla u_{0}$ uniformly on compact subsets of $\left\{u_{0}>0\right\}$,

(4) $\nabla u_{k} \rightarrow \nabla u_{0}$ a.e. in $\mathbb{R}^{N}$,

(5) If $x_{k} \in \partial\{u>0\}$, then $0 \in \partial\left\{u_{0}>0\right\}$,

(6) $\Delta_{p\left(x_{0}\right)} u_{0}=0$ in $\left\{u_{0}>0\right\}$,

(7) $u_{0}$ is Lipschitz continuous and satisfies property (2) of Definition 2.2 in $\mathbb{R}^{N}$ with the same constants as $u$ in a ball $B_{\rho_{0}}\left(x_{0}\right) \subset \subset \Omega$.

Proof. The proof follows with similar ideas to those in [1], 4.7 and [2], pp. 19-20. We here use that $\Delta_{p_{k}(x)} u_{k}=f_{k}$ in $\left\{u_{k}>0\right\}$, where $p_{k}(x)=p\left(x_{k}+\rho_{k} x\right)$ and $f_{k}(x)=\rho_{k} f\left(x_{k}+\rho_{k} x\right)$ satisfy $p_{k} \rightarrow p\left(x_{0}\right)$ and $f_{k} \rightarrow 0$ uniformly on compact sets of $\mathbb{R}^{N}$. This implies that $\nabla u_{k}$ are uniformly Hölder continuous on compact subsets of $\left\{u_{0}>0\right\}$. (Notice that some of these arguments were already employed in the proof of Lemma 2.4).

We will next prove an identification result for the function $q_{u}$ given in Theorem 2.1, which holds at points $x_{0} \in \partial_{\text {red }}\{u>0\}$ that are Lebesgue points of the function $q_{u}$ and are such that

$$
\limsup _{r \rightarrow 0} \frac{\mathcal{H}^{N-1}\left(\partial\{u>0\} \cap B\left(x_{0}, r\right)\right)}{\mathcal{H}^{N-1}\left(B^{\prime}\left(x_{0}, r\right)\right)} \leq 1 .
$$

(Here $\left.B^{\prime}\left(x_{0}, r\right)=\left\{x^{\prime} \in \mathbb{R}^{N-1} /\left|x^{\prime}\right|<r\right\}\right)$.

Notice that under our assumptions, $\mathcal{H}^{N-1}-$ a.e. point in $\partial_{\text {red }}\{u>0\}$ satisfies (2.8) (see Theorem 4.5.6(2) in [15]).

Lemma 2.6. Assume that $u$ satisfies hypotheses (1), (2) and (3) of Definition 2.2. Then, $q_{u}\left(x_{0}\right)=$ $\lambda^{*}\left(x_{0}\right)^{p\left(x_{0}\right)-1}$ for $\mathcal{H}^{N-1}$ a.e. $x_{0} \in \partial_{\text {red }}\{u>0\}$.

Proof. If $u$ satisfies (3) of Definition 2.2, take $x_{0} \in \partial_{\text {red }}\{u>0\}$ such that

$$
u(x)=\lambda^{*}\left(x_{0}\right)\left\langle x-x_{0}, \nu\left(x_{0}\right)\right\rangle^{-}+o\left(\left|x-x_{0}\right|\right),
$$

where $\nu\left(x_{0}\right)$ is the exterior unit normal at $x_{0}$ in the measure theoretic sense. We assume $\nu\left(x_{0}\right)=e_{N}$. Take $\rho_{k} \rightarrow 0$ and $u_{k}(x)=\frac{1}{\rho_{k}} u\left(x_{0}+\rho_{k} x\right)$. If $\xi \in C_{0}^{\infty}(\Omega)$ we have

$$
-\int_{\{u>0\}}|\nabla u|^{p(x)-2} \nabla u \cdot \nabla \xi d x-\int_{\{u>0\}} f \xi d x=\int_{\partial\{u>0\}} q_{u}(x) \xi d \mathcal{H}^{N-1},
$$


and if we replace $\xi$ by $\xi_{k}(x)=\rho_{k} \xi\left(\frac{x-x_{0}}{\rho_{k}}\right)$ with $\xi \in C_{0}^{\infty}\left(B_{R}\right), k \geq k_{0}$ and we change variables, we obtain

$$
-\int_{\left\{u_{k}>0\right\}}\left|\nabla u_{k}\right|^{p_{k}(x)-2} \nabla u_{k} \cdot \nabla \xi d x-\int_{\left\{u_{k}>0\right\}} f_{k} \xi d x=\int_{\partial\left\{u_{k}>0\right\}} q_{u}\left(x_{0}+\rho_{k} x\right) \xi d \mathcal{H}^{N-1},
$$

where $p_{k}(x)=p\left(x_{0}+\rho_{k} x\right)$ and $f_{k}(x)=\rho_{k} f\left(x_{0}+\rho_{k} x\right)$. From Lemma 2.5, it follows that, for a subsequence, $u_{k} \rightarrow u_{0}$ uniformly on compact sets of $\mathbb{R}^{N}$, with $u_{0}(x)=\lambda^{*}\left(x_{0}\right) x_{N}^{-}$and moreover, $\left|\nabla u_{k}\right|^{p_{k}(x)-2} \nabla u_{k} \rightarrow\left|\nabla u_{0}\right|^{p_{0}-2} \nabla u_{0}$ a.e. in $\mathbb{R}^{N}$, with $p_{0}=p\left(x_{0}\right)$. Thus,

$$
-\int_{\left\{u_{k}>0\right\}}\left|\nabla u_{k}\right|^{p_{k}(x)-2} \nabla u_{k} \cdot \nabla \xi d x-\int_{\left\{u_{k}>0\right\}} f_{k} \xi d x \rightarrow-\int_{\left\{x_{N}<0\right\}}\left|\nabla u_{0}\right|^{p_{0}-2} \nabla u_{0} \cdot \nabla \xi d x .
$$

We now let

$$
\xi(x)=\min \left(2\left(1-\left|x_{N}\right|\right)^{+}, 1\right) \eta\left(x_{1}, \ldots, x_{N-1}\right),
$$

for $\left|x_{N}\right| \leq 1$ and $\xi=0$ otherwise, where $\eta \in C_{0}^{\infty}\left(B_{r}^{\prime}\right)$, (where $B_{r}^{\prime}$ is a ball $(N-1)$ dimensional with radius $r$ ) and $\eta \geq 0$. Then, if $x_{0}$ is a Lebesgue point of $q_{u}$ satisfying (2.8), we proceed as in [1], p.121 and we get

$$
\int_{\partial\left\{u_{k}>0\right\}} q_{u}\left(x_{0}+\rho_{k} x\right) \xi d \mathcal{H}^{N-1} \rightarrow q_{u}\left(x_{0}\right) \int_{\left\{x_{N}=0\right\}} \xi d \mathcal{H}^{N-1} .
$$

As $\nabla u_{0}=-\lambda^{*}\left(x_{0}\right) e_{N} \chi_{\left\{x_{N}<0\right\}}$, it follows that

$$
\lambda^{*}\left(x_{0}\right)^{p_{0}-1} \int_{B_{r}^{\prime}} \xi\left(x^{\prime}, 0\right) d \mathcal{H}^{N-1}=q_{u}\left(x_{0}\right) \int_{B_{r}^{\prime}} \xi\left(x^{\prime}, 0\right) d \mathcal{H}^{N-1} .
$$

Thus, we deduce that for $\mathcal{H}^{N-1}$-almost every point $x_{0} \in \partial_{\text {red }}\{u>0\}, q_{u}\left(x_{0}\right)=\lambda^{*}\left(x_{0}\right)^{p\left(x_{0}\right)-1}$.

\section{Flat FREE BOUNDARY POINTS}

In this section we study the behavior of weak solutions to the free boundary problem $P\left(f, p, \lambda^{*}\right)$ near "flat" free boundary points.

Throughout the section we assume, unless otherwise stated, that $f$ is bounded, $p(x)$ is Lipschitz continuous and $\lambda^{*}(x)$ is Hölder continuous.

As in previous papers, we start by defining the flatness classes.

Definition 3.1. Let $0<\sigma_{1}, \sigma_{2} \leq 1, \tau>0$. We say that $u$ belongs to the class $F\left(\sigma_{1}, \sigma_{2} ; \tau\right)$ in $B_{\rho}\left(x_{0}\right)$ in direction $\nu$ with power $p(x)$, slope $\lambda^{*}(x)$ and right hand side $f(x)$ if $u$ is a weak solution to the free boundary problem $P\left(f, p, \lambda^{*}\right)$ in $B_{\rho}\left(x_{0}\right), x_{0} \in \partial\{u>0\}$ and

(1) $u(x)=0$ if $\left\langle x-x_{0}, \nu\right\rangle \geq \sigma_{1} \rho, x \in B_{\rho}\left(x_{0}\right)$,

(2) $u(x) \geq-\lambda^{*}\left(x_{0}\right)\left(\left\langle x-x_{0}, \nu\right\rangle+\sigma_{2} \rho\right)$ if $\left\langle x-x_{0}, \nu\right\rangle \leq-\sigma_{2} \rho, x \in B_{\rho}\left(x_{0}\right)$,

(3) $|\nabla u| \leq \lambda^{*}\left(x_{0}\right)(1+\tau)$ in $B_{\rho}\left(x_{0}\right)$.

After a rotation and a translation we may assume that $x_{0}=0$ and $\nu=e_{N}$. We will not explicitly mention the direction of flatness when $\nu=e_{N}$.

We may further reduce the analysis to the unit ball by the following transformations:

$$
\bar{u}(x)=\frac{u(\rho x)}{\rho}, \quad \bar{p}(x)=p(\rho x), \quad \overline{\lambda^{*}}(x)=\lambda^{*}(\rho x), \quad \bar{f}(x)=\rho f(\rho x) .
$$

Then, if $u \in F\left(\sigma_{1}, \sigma_{2} ; \tau\right)$ in $B_{\rho}$ with power $p$, slope $\lambda^{*}$ and right hand side $f$, there holds that $\bar{u} \in F\left(\sigma_{1}, \sigma_{2} ; \tau\right)$ in $B_{1}$ with power $\bar{p}$, slope $\bar{\lambda}^{*}$ and right hand side $\bar{f}$. 
Observe that, if $1<p_{\min } \leq p(x) \leq p_{\max }<\infty, 0<\lambda_{\min } \leq \lambda^{*}(x) \leq \lambda_{\max }<\infty, p \in$ Lip with $|\nabla p| \leq L_{1}, \lambda^{*} \in C^{\alpha^{*}}$ with $\left[\lambda^{*}\right]_{C^{*}\left(B_{\rho}\right)} \leq C^{*}$ and $f \in L^{\infty}\left(B_{\rho}\right)$ with $|f| \leq L_{2}$, there holds that $\bar{p}, \lambda^{*}$ and $\bar{f}$ are in similar spaces in $B_{1}$ and $1<p_{\min } \leq \bar{p}(x) \leq p_{\max }<\infty, 0<\lambda_{\min } \leq \bar{\lambda}^{*}(x) \leq \lambda_{\max }<\infty$, $|\nabla \bar{p}| \leq L_{1} \rho,|\bar{f}| \leq L_{2} \rho$ and $\left[\bar{\lambda}^{*}\right]_{C^{\alpha^{*}\left(B_{1}\right)}} \leq C^{*} \rho^{\alpha^{*}}$.

The first lemma states that, if $u$ vanishes for $x_{N} \geq \sigma$, there holds that, in a smaller ball, $u$ is above a hyperplane for $x_{N} \leq-\varepsilon$.

Lemma 3.1. Let $p \in \operatorname{Lip}\left(B_{1}\right), \lambda^{*} \in C^{\alpha^{*}}\left(B_{1}\right), f \in L^{\infty}\left(B_{1}\right)$ with $|\nabla p| \leq L_{1} \rho,|f| \leq L_{2} \rho$, $\left[\lambda^{*}\right]_{C^{\alpha^{*}}\left(B_{1}\right)} \leq C^{*} \rho^{\alpha^{*}}$ and $C^{*} \rho^{\alpha^{*}} \leq \lambda^{*}(0) \sigma$. Let $u \in F(\sigma, 1 ; \sigma)$ in $B_{1}$ with power $p$, slope $\lambda^{*}$ and rhs $f$.

Let $0<\varepsilon \leq 1 / 2$ and $\frac{1}{2} \leq R<1$. There exists $\sigma_{0}=\sigma_{0}\left(\varepsilon, N, R, p_{\min }, p_{\max }, \lambda_{\min }, \lambda_{\max }, L_{1}, L_{2}, C^{*}\right)$ such that if $\sigma \leq \sigma_{0}$ there holds that $u \in F(\sigma / R, \varepsilon ; \sigma)$ in $B_{R}$ with the same power, slope and rhs.

Proof. We follow the construction of [2] with the variation of [8]. In this paper, we consider an arbitrary $R$ instead of $R=1 / 2$ in order to pursue the argument in the next steps.

Let $R^{\prime}=R+(1-R) / 4$. As in these papers, we will prove that, for every $0<r \leq(1-R) / 8$ there exists $\sigma_{0}=\sigma_{0}\left(r, R, p_{\min }, p_{\max }, \lambda_{\min }, \lambda_{\max }, L_{1}, L_{2}, C^{*}\right)$ such that for $\sigma \leq \sigma_{0}$,

$$
u(\xi) \geq \lambda^{*}(0)\left[-\xi_{N}-4 r\right] \text { for } \xi \in \partial B_{R^{\prime}} \text { with } \xi_{N} \leq-\frac{(1-R)}{4} .
$$

Then, integrating along vertical lines a distance at most $R^{\prime}$ and using that $|\nabla u| \leq \lambda^{*}(0)(1+\sigma)$, we get

$$
\begin{aligned}
u\left(\xi^{\prime}, \xi_{N}+\alpha\right) & \geq u(\xi)-\lambda^{*}(0)(1+\sigma) \alpha \\
& \geq \lambda^{*}(0)\left[-\left(\xi_{N}+\alpha\right)-4 r-R^{\prime} \sigma\right] \\
& \geq \lambda^{*}(0)\left[-\left(\xi_{N}+\alpha\right)-\varepsilon R\right]
\end{aligned}
$$

if $0 \leq \alpha \leq R^{\prime}, r=\min \left\{\frac{R \varepsilon}{8}, \frac{1-R}{8}\right\}$ and $\sigma \leq \min \left\{\frac{R \varepsilon}{R+1}, \sigma_{0}\right\}$.

This implies that, for $|x|<R, x_{N} \leq-R \varepsilon$,

$$
u(x) \geq-\lambda^{*}(0)\left(x_{N}+R \varepsilon\right) .
$$

So that $u \in F(\sigma / R, \varepsilon ; \sigma)$ in $B_{R}$ with power $p$, slope $\lambda^{*}$ and rhs $f$, and the lemma will be proved.

In order to prove (3.2), we will show that, once we fix $0<r \leq \frac{(1-R)}{8}$ there exists $\kappa>0$ such that, for every $\xi \in \partial B_{R^{\prime}}$ with $\xi_{N} \leq-(1-R) / 4$, there exists $x_{\xi} \in \partial B_{r}(\xi)$ such that

$$
u\left(x_{\xi}\right) \geq-\lambda^{*}(0)(1-\kappa \sigma) x_{\xi_{N}} .
$$

Then, by using again that $|\nabla u| \leq \lambda^{*}(0)(1+\sigma)$,

$$
\begin{aligned}
u(\xi) & \geq u\left(x_{\xi}\right)-\lambda^{*}(0)(1+\sigma) r \geq \lambda^{*}(0)\left[-(1-\kappa \sigma) x_{\xi_{N}}-(1+\sigma) r\right] \\
& \geq \lambda^{*}(0)\left[-\xi_{N}-r-\kappa \sigma-2 r\right] \geq \lambda^{*}(0)\left[-\xi_{N}-4 r\right]
\end{aligned}
$$

if $\sigma \leq \frac{r}{\kappa}$, that is, we get (3.2).

The existence of a point $x_{\xi}$ satisfying (3.3) is done by assuming that such a point does not exist and getting a contradiction if $\kappa$ is large depending on $r, R$ and the constants in the structure conditions. The inequality that will allow to get this contradiction will be achieved if $\sigma$ is small depending on the same parameters. Such inequality comes from the construction of two barriers in the following way: 
Let $\eta \in C_{0}^{\infty}\left(B_{1}^{\prime}\right)$ given by

$$
\eta(y)=\left\{\begin{array}{lll}
\exp \left(-\frac{9|y|^{2}}{1-9|y|^{2}}\right) & \text { if } & |y|<\frac{1}{3} \\
0 & \text { if } & |y| \geq \frac{1}{3}
\end{array}\right.
$$

Let $s \geq 0$ be maximal such that

$$
B_{1} \cap\{u>0\} \subset D:=\left\{x \in B_{1}: x_{N}<\sigma-s \eta\left(x^{\prime}\right)\right\} .
$$

Then, as $0 \in \partial\{u>0\}$ there holds that $s \leq \sigma$.

First, we let $v \in W^{1, p(\cdot)}\left(D \backslash \overline{B_{r}(\xi)}\right)$ be the solution to

$$
\begin{cases}\Delta_{p(x)} v=-L_{2} \rho & \text { in } D \backslash \overline{B_{r}(\xi),} \\ v=0 & \text { on } \partial D \cap B_{1}, \\ v=\lambda^{*}(0)(1+\sigma)\left(\sigma-x_{N}\right) & \text { on } \partial D \backslash B_{1}, \\ v=-\lambda^{*}(0)(1-\kappa \sigma) x_{N} & \text { on } \quad \partial B_{r}(\xi) .\end{cases}
$$

Since the boundary datum coincides with $\lambda^{*}(0)(1+\sigma)\left(\sigma-x_{N}-s \eta\left(x^{\prime}\right)\right)$ on $\partial D$, it has an extension $\phi \in W^{1, \infty}\left(D \backslash \overline{B_{r}(\xi)}\right)$ and therefore the solution $v$ exists by a minimization argument in $\phi+$ $W_{0}^{1, p(\cdot)}\left(D \backslash \overline{B_{r}(\xi)}\right)$.

As we are assuming that (3.3) does not hold for any $x_{\xi} \in \partial B_{r}(\xi)$ and, since $u=0$ if $x \in \partial D \cap B_{1}$ and $|\nabla u| \leq \lambda^{*}(0)(1+\sigma)$, there holds that $u \leq v$ on $\partial\left(D \backslash \overline{B_{r}(\xi)}\right)$. Now, recalling Lemma 2.1, we get $\Delta_{p(x)} u \geq f \chi_{\{u>0\}} \geq-L_{2} \rho$, then comparison of weak sub- and super-solutions gives

$$
u \leq v \quad \text { in } \quad D \backslash \overline{B_{r}(\xi)}
$$

Now, let $z \in \partial D \cap \partial\{u>0\} \cap\left\{\left|z^{\prime}\right|<1 / 3\right\}$. Then, there exists a ball $B$ contained in $\{u=0\}$ such that $z \in \partial B$. By the definition of weak solution and, since $\lambda^{*}(z) \geq \lambda^{*}(0)-C^{*} \rho^{\alpha^{*}}|z|^{\alpha^{*}} \geq \lambda^{*}(0)(1-\sigma)$, we deduce that

$$
\lambda^{*}(0)(1-\sigma) \leq \lambda^{*}(z) \leq \limsup _{\substack{x \rightarrow z \\ u(x)>0}} \frac{u(x)}{\operatorname{dist}(x, B)} \leq|\nabla v(z)| .
$$

We will get a contradiction once we find a barrier from above for $v$ in the form $w=v_{1}-\kappa \sigma v_{2}$ with $\left|\nabla v_{1}\right| \leq \lambda^{*}(0)\left(1+C_{3} \sigma\right),\left|\nabla v_{2}\right| \geq c \lambda^{*}(0)>0, v_{1}>0, v_{2}>0$ close to $z$ and $v_{1}=v_{2}=0$ on $\partial D \cap B_{1}$ close to $z$. In fact, if such a barrier $w$ exists, by (3.5) there holds that

$$
\lambda^{*}(0)(1-\sigma) \leq|\nabla v(z)| \leq|\nabla w(z)|=\left|\nabla v_{1}(z)\right|-\kappa \sigma\left|\nabla v_{2}(z)\right| \leq \lambda^{*}(0)\left[1+C_{3} \sigma-c \kappa \sigma\right]
$$

and this is a contradiction if $\kappa$ is large depending only on $C_{3}$ and $c$. Since the constants $C_{3}$ and $c$ will depend only on $r, R, p_{\min }, p_{\max }, \lambda_{\min }, \lambda_{\max }, L_{1}, L_{2}$ and $C^{*}$, the lemma will be proved.

As in [8] and [16], the idea of the construction of $v_{1}$ and $v_{2}$ is that they will be such that $w=v_{1}-\kappa \sigma v_{2}$ will satisfy

$$
\frac{\lambda^{*}(0)}{2} \leq|\nabla w| \leq 2 \lambda^{*}(0)
$$

if $\sigma$ is small depending on those constants. Then,

$$
\Delta_{p(x)} w=|\nabla w|^{p(x)-2}\left[\sum_{i j} b_{i j}(x) w_{x_{i} x_{j}}+\sum_{j} b_{j}(x) w_{x_{j}}\right]
$$


with $b_{i j}=\delta_{i j}+(p(x)-2) \frac{w_{x_{i}} w_{x_{j}}}{|\nabla w|^{2}}$ and $b_{j}=p_{x_{j}} \log |\nabla w|$. There holds that

$$
\beta_{1}|\nu|^{2} \leq \sum_{i j} b_{i j} \nu_{i} \nu_{j} \leq \beta_{2}|\nu|^{2} \quad \forall \nu \in \mathbb{R}^{N}
$$

with $\beta_{1}=\min \left\{1, p_{\min }-1\right\}, \beta_{2}=\max \left\{1, p_{\max }-1\right\}$ and, with $\Lambda=\max \left\{\left|\log \lambda_{\min }\right|,\left|\log \lambda_{\max }\right|\right\}+\log 2$, $b=\left(b_{1}, \cdots, b_{N}\right)$,

$$
|b| \leq \Lambda L_{1} \rho \leq \frac{\Lambda L_{1} \lambda_{\max }}{C^{*}} \sigma=C_{0} \sigma,
$$

if $\sigma \leq \frac{C^{*}}{\lambda_{\max }}$, with $C_{0}=\frac{\Lambda L_{1} \lambda_{\max }}{C^{*}}$.

Thus, the idea is to construct $v_{1}$ in such a way that

$$
\frac{2}{3} \lambda^{*}(0) \leq\left|\nabla v_{1}\right| \leq \frac{3}{2} \lambda^{*}(0)
$$

and

$$
\mathcal{T} v_{1} \leq-S^{-1} L_{2} \frac{\lambda_{\max }}{C^{*}} \sigma=-M \sigma \quad \text { in } \quad D,
$$

with $S=\min \left\{\left(\frac{\lambda_{\min }}{2}\right)^{p_{\min }-2},\left(\frac{\lambda_{\min }}{2}\right)^{p_{\max }-2},\left(2 \lambda_{\max }\right)^{p_{\min }-2},\left(2 \lambda_{\max }\right)^{p_{\max }-2}\right\}$ for any operator

$$
\mathcal{T}=\sum_{i j} b_{i j}(x) \partial_{x_{i} x_{j}}+\sum_{j} b_{j}(x) \partial_{x_{j}}
$$

with $\left\{b_{i j}\right\}$ satisfying (3.7) with $\beta_{1}=\min \left\{1, p_{\min }-1\right\}, \beta_{2}=\max \left\{1, p_{\max }-1\right\}$ and $\left\{b_{j}\right\}$ satisfying

$$
|b| \leq C_{0} \sigma
$$

with $C_{0}$ the constant in (3.8).

Then, $v_{2}$ will be a function satisfying

$$
\mathcal{T} v_{2} \geq 0 \quad \text { in } \quad \widetilde{D} \backslash B_{r}(\xi)
$$

for any such an operator $\mathcal{T}$ with

$$
0<c \lambda^{*}(0) \leq\left|\nabla v_{2}\right| \leq C \lambda^{*}(0)
$$

for some constants $c, C$ depending only on $R, r$. Here $\widetilde{D}$ is a smooth domain contained in $D$ and containing $D \backslash B_{(1-R) / 10}\left(\partial B_{1}^{\prime} \times\{0\}\right)$. In this way, once we fix $\kappa>0$ there holds that $w$ satisfies (3.6) if $\sigma$ is small and therefore,

$$
\Delta_{p(x)} w \leq-L_{2} \rho=\Delta_{p(x)} v \quad \text { in } \quad \widetilde{D} \backslash B_{r}(\xi) .
$$

The functions $v_{1}$ and $v_{2}$ are also constructed in such a way that $w \geq v$ on $\partial\left(\widetilde{D} \backslash B_{r}(\xi)\right)$.

As in the previously cited papers, we let

$$
d_{1}(x)=-x_{N}+\sigma-s \eta\left(x^{\prime}\right) \quad \text { and } \quad v_{1}(x)=\lambda^{*}(0) \frac{\gamma_{1}}{\mu_{1}}\left(1-e^{-\mu_{1} d_{1}(x)}\right) \quad \text { in } \quad D
$$

with $\mu_{1}=C_{1} \sigma$ and $\gamma_{1}=1+C_{2} \sigma$. Then, $\left|\nabla v_{1}\right| \leq \lambda^{*}(0)(1+C \sigma)\left(1+C_{2} \sigma\right)$ with $C$ depending only on $\eta$ (in particular, $\left|\nabla v_{1}\right| \leq \lambda^{*}(0)\left(1+C_{3} \sigma\right)$ with $C_{3}$ depending only on $C_{2}$ and $\eta$ ). Moreover, $D_{x_{i} x_{j}} v_{1}=\lambda^{*}(0) \gamma_{1} e^{-\mu_{1} d_{1}}\left[D_{x_{i} x_{j}} d_{1}-\mu_{1} d_{1 x_{i}} d_{1 x_{j}}\right]$. Thus,

$$
\begin{aligned}
\mathcal{T} v_{1} & \leq \gamma_{1} e^{-\mu_{1} d_{1}}\left[N^{2} \lambda_{\max } \beta_{2}\left\|D^{2} \eta\right\|_{\left.L^{\infty} \sigma-\lambda_{\min } \beta_{1} \mu_{1}+\lambda_{\max } C_{0}\left(1+C_{3} \sigma\right) \sigma\right]}\right. \\
& \leq\left[2 N^{2} \lambda_{\max } \beta_{2}\left\|D^{2} \eta\right\|_{L^{\infty}}+4 \lambda_{\max } C_{0}-e^{-2} C_{1} \lambda_{\min } \beta_{1}\right] \sigma \\
& \leq-M \sigma
\end{aligned}
$$


if $\sigma \leq \sigma\left(C_{1}, C_{2}, C_{3}\right)$ and $C_{1} \geq C_{1}\left(\lambda_{\min }, \lambda_{\max }, \beta_{1}, \beta_{2}, C_{0}, M\right) . C_{1}$ is fixed from now on.

On the other hand,

$$
\frac{2}{3} \lambda^{*}(0) \leq \lambda^{*}(0)\left(1+C_{2} \sigma\right) e^{-C_{1} \sigma(1+\sigma)} \leq\left|\nabla v_{1}\right| \leq \lambda^{*}(0)\left(1+C_{3} \sigma\right) \leq \frac{3}{2} \lambda^{*}(0)
$$

if $\sigma \leq \sigma\left(C_{1}, C_{2}, C_{3}\right)$.

The constant $C_{2}$ (and therefore also $C_{3}$ ) will be fixed now in order to guaranty that $w \geq v$ on the boundary of $D \backslash B_{r}(\xi)$.

First, on $\partial D \cap B_{1}$ we have $v_{1}=0$.

Observe that

$$
v_{1}(x) \geq \lambda^{*}(0)\left(1+C_{2} \sigma\right) e^{-2 C_{1} \sigma} d_{1} \geq \lambda^{*}(0)\left(1+\frac{C_{2}}{2} \sigma\right) d_{1} \geq \lambda^{*}(0)(1+4 \sigma) d_{1}
$$

if $C_{2} \geq 8$ and $\sigma \leq \sigma\left(C_{1}, C_{2}\right)$.

Now, on $\partial D \backslash B_{1}$ we consider two cases:

(a) $\left|x^{\prime}\right| \geq \frac{1}{3}$. Then, $\eta\left(x^{\prime}\right)=0$ and $d_{1}=\sigma-x_{N}$. Thus,

$$
v_{1}(x) \geq \lambda^{*}(0)(1+\sigma)\left(\sigma-x_{N}\right) .
$$

(b) $\left|x^{\prime}\right|<\frac{1}{3}$. Then, $\left|x_{N}\right|>\sqrt{\frac{2}{3}}$ and

$$
\begin{aligned}
v_{1}(x) & \geq \lambda^{*}(0)(1+4 \sigma)\left(\sigma-x_{N}-s \eta\left(x^{\prime}\right)\right) \\
& \geq \lambda^{*}(0)(1+\sigma)\left(\sigma-x_{N}\right)+\lambda^{*}(0)\left[3\left(\sigma-x_{N}\right)-(1+4 \sigma)\right] \sigma \\
& \geq \lambda^{*}(0)(1+\sigma)\left(\sigma-x_{N}\right)+\lambda^{*}(0)[\sqrt{6}-(1+4 \sigma)] \sigma \\
& \geq \lambda^{*}(0)(1+\sigma)\left(\sigma-x_{N}\right)
\end{aligned}
$$

if $C_{2} \geq 8, \sigma \leq \sigma\left(C_{1}, C_{2}\right)$ and $\sqrt{6}-(1+4 \sigma) \geq 0$.

Finally, if $x \in \partial B_{r}(\xi)$ and, since $r \leq \frac{(1-R)}{8}$, there holds that $x_{N}<0$, so that

$$
\begin{aligned}
v_{1}(x) & \geq \lambda^{*}(0)(1+4 \sigma)\left(\sigma-x_{N}-s \eta\left(x^{\prime}\right)\right) \\
& =\lambda^{*}(0)\left[-x_{N}+(1+4 \sigma)\left(\sigma-s \eta\left(x^{\prime}\right)\right)-4 \sigma x_{N}\right] \\
& \geq-\lambda^{*}(0) x_{N} .
\end{aligned}
$$

Therefore, we can fix $C_{2}=8$ for our construction of $v_{1}$.

Now, we construct $v_{2}$ in $\widetilde{D} \backslash B_{r}(\xi)$ with $\widetilde{D}$ as described above. We take $d_{2}$ such that

$$
d_{2} \in C^{2}\left(\widetilde{\widetilde{D} \backslash B_{r}(\xi)}\right), \quad d_{2}=0 \text { on } \partial \widetilde{D}, \quad 0 \leq d_{2} \leq 1 \text { in } \widetilde{D} \backslash B_{r}(\xi)
$$

and, moreover

$$
0<\tilde{c} \leq\left|\nabla d_{2}\right| \leq \tilde{C} \quad \text { in } \quad \widetilde{D} \backslash B_{r}(\xi)
$$

with $\tilde{C}, \tilde{c}$ depending only on $r, R$.

Then, we take

$$
v_{2}(x)=\lambda^{*}(0) \frac{\gamma_{2}}{\mu_{2}}\left(e^{\mu_{2} d_{2}(x)}-1\right) .
$$

First, we fix $\mu_{2}$. Then, $\gamma_{2}$ is fixed so that $v_{2} \leq \frac{(1-R)}{8} \lambda^{*}(0)$, that is,

$$
\gamma_{2}=\frac{(1-R)}{8} \frac{\mu_{2}}{\left(e^{\mu_{2}}-1\right)} \text {. }
$$


Thus, there exist constants depending only on $\tilde{c}, \tilde{C}, \mu_{2}, R$ such that

$$
0<c \lambda^{*}(0) \leq\left|\nabla v_{2}\right| \leq C \lambda^{*}(0) \text {. }
$$

Now, we fix $\mu_{2}$ so that $\mathcal{T} v_{2} \geq 0$ in $\widetilde{D} \backslash B_{r}(\xi)$ for any operator $\mathcal{T}$ as above.

There holds

$$
\mathcal{T} v_{2} \geq \gamma_{2}\left[\mu_{2} \lambda_{\min } \beta_{1} \tilde{c}^{2}-\beta_{2} \lambda_{\max }\left\|D^{2} d_{2}\right\|_{L^{\infty}}-\tilde{C} C_{0} \sigma \lambda_{\max }\right] \geq 0
$$

if $\mu_{2} \geq \mu_{2}\left(\lambda_{\min }, \lambda_{\max }, \beta_{1}, \beta_{2}, \tilde{c}, \tilde{C}, C_{0}\right)$. (Recall that $\tilde{c}$ and $\tilde{C}$ depend only on $r, R$ ).

Now, in order to finish our proof we need to see that $w=v_{1}-\kappa \sigma v_{2} \geq v$ in $\widetilde{D} \backslash B_{r}(\xi)$. For this purpose, it only remains to show that the inequality holds on $\partial B_{r}(\xi)$, that is, we have to prove that

$$
w(x)=v_{1}(x)-\kappa \sigma v_{2}(x) \geq-\lambda^{*}(0)(1-\kappa \sigma) x_{N} \quad \text { on } \quad \partial B_{r}(\xi) .
$$

Recall that $v_{2} \leq \frac{(1-R)}{8} \lambda^{*}(0)$. Thus,

$$
w(x)=v_{1}(x)-\kappa \sigma v_{2}(x) \geq \lambda^{*}(0)\left(-x_{N}-\frac{(1-R)}{8} \kappa \sigma\right) \geq-\lambda^{*}(0)(1-\kappa \sigma) x_{N}
$$

since $x_{N} \leq-\frac{(1-R)}{8}$ for $x \in \partial B_{r}(\xi)$.

And we get a contradiction as discussed above.

The following lemma gives a control of the gradient of $u$ from below on compact sets of $B_{1}^{-}$.

Lemma 3.2. Let $p, \lambda^{*}, f, \rho, u$ as in Lemma 3.1. For every $\varepsilon, \delta>0, \frac{1}{2} \leq R<1$, there exists $\sigma_{0}$ depending on $\varepsilon, N, \delta, R, p_{\min }, p_{\max }, \lambda_{\min }, \lambda_{\max }, L_{1}, L_{2}, C^{*}$ such that, if $\sigma \leq \sigma_{0}$ there holds that

$$
|\nabla u| \geq \lambda^{*}(0)(1-\delta) \quad \text { in } \quad B_{R} \cap\left\{x_{N} \leq-\varepsilon\right\} .
$$

Proof. The proof is entirely similar to the one of Lemma 6.6 in [8]. Let $R<R^{\prime}<1$. As in [8] we use a contradiction argument. In our case by Lemma 3.1, we have that the functions $u_{k} \in F\left(\frac{1}{k}, 1 ; \frac{1}{k}\right)$ in $B_{1}$ satisfy

$$
\Delta_{p_{k}(x)} u_{k}=f_{k} \quad \text { in } \quad \mathcal{K} \subset \subset B_{R^{\prime}}^{-}
$$

if $k$ is large depending on $\mathcal{K}$. Here $\left|f_{k}\right| \leq L_{2} \rho_{k}, 1<p_{\min } \leq p_{k}(x) \leq p_{\max }<\infty,\left|\nabla p_{k}\right| \leq L_{1} \rho_{k}$ and $C^{*} \rho_{k} \alpha^{*} \leq \frac{\lambda_{k}^{*}(0)}{k}$. Thus, by the regularity estimates in [14], for a subsequence, $\nabla u_{k}$ converges uniformly on compact subsets of $B_{R^{\prime}}^{-}$. And the proof follows as in [8].

Now we can prove one of the main results that states that, flatness to the right ( $u$ vanishing for $\left.x_{N} \geq \sigma\right)$ implies flatness to the left in a smaller ball.

Proposition 3.1. Let $p, \lambda^{*}, f, \rho, u$ as in Lemma 3.1. Let $1 / 2 \leq R<1$. There exist $\sigma_{0}=\sigma_{0}\left(N, R, p_{\min }, p_{\max }, \lambda_{\min }, \lambda_{\max }, L_{1}, L_{2}, C^{*}\right), C_{0}=C_{0}\left(N, R, p_{\min }, p_{\max }, \lambda_{\min }, \lambda_{\max }, L_{1}, L_{2}, C^{*}\right)$ such that, if $\sigma \leq \sigma_{0}$ there holds that $u \in F\left(\sigma / R, C_{0} \sigma ; \sigma\right)$ in $B_{R}$ with the same power, slope and rhs.

Proof. The proof follows as the one of Theorem 6.3 in [8]. We let $R^{\prime}=R+(1-R) / 4$ and $R^{\prime \prime}=R+(1-R) / 2$. In our case, since $|\nabla u| \geq \frac{\lambda^{*}(0)}{2}$ in $\overline{B_{R^{\prime \prime}}} \cap\left\{x_{N} \leq-(1-R) / 8\right\}$ if $\sigma$ is small and $|\nabla u| \leq 2 \lambda^{*}(0)$, there holds that $u$ satisfies

$$
\mathcal{T} u=|\nabla u|^{2-p(x)} f(x) \quad \text { in } \quad B_{R^{\prime \prime}} \cap\left\{x_{N}<-(1-R) / 8\right\}
$$

for an operator as the one considered in Lemma 3.1.

Then, as in [8] (see also [2]) we take

$$
w(x)=\lambda^{*}(0)(1+\sigma)\left(\sigma-x_{N}\right)-u(x)
$$


that satisfies

$$
\mathcal{T} w=-\lambda^{*}(0)(1+\sigma) b_{N}-|\nabla u|^{2-p(x)} f(x) \quad \text { in } \quad B_{R^{\prime \prime}} \cap\left\{x_{N}<-\frac{(1-R)}{8}\right\}
$$

and, using that $w \geq 0$ in $B_{1} \cap\left\{x_{N} \leq \sigma\right\}$, taking $\xi \in \partial B_{R^{\prime}} \cap\left\{x_{N} \leq-(1-R) / 4\right\}$, applying Harnack inequality in $B_{(1-R) / 8}(\xi)$ and using that the right hand side is bounded by $C \sigma$ for a constant $C$ depending only on $R, p_{\min }, p_{\max }, \lambda_{\min }, \lambda_{\max }, L_{1}, L_{2}$ and $C^{*}$ we get, as in $[2,8]$,

$$
w(\xi) \leq \widetilde{C} \lambda^{*}(0) \sigma .
$$

Then, the proof follows as in [8].

Finally, we can improve on the control of the gradient.

Lemma 3.3. Let $p, \lambda^{*}, f, \rho, u$ as in Lemma 3.1. For every $1 / 2 \leq R<1,0<\delta<1$ there exists $\sigma_{\delta, R}$ and $C_{\delta, R}$ depending also on $N, p_{\min }, p_{\max }, \lambda_{\min }, \lambda_{\max }, L_{1}, L_{2}, C^{*}$ such that, if $\sigma \leq \sigma_{\delta, R}$ there holds that

$$
|\nabla u| \geq \lambda^{*}(0)(1-\delta) \quad \text { in } \quad B_{R} \cap\left\{x_{N} \leq-C_{\delta, R} \sigma\right\} .
$$

Proof. It follows exactly as the proof of Theorem 6.4 in [8].

Observe that the scalings $\bar{p}_{k}(x)=p_{k}\left(y_{k}+2 d_{k} x\right), \bar{\lambda}_{k}^{*}(x)=\lambda_{k}^{*}\left(y_{k}+2 d_{k} x\right)$ and $\bar{f}_{k}(x)=2 d_{k} f_{k}\left(y_{k}+\right.$ $\left.2 d_{k} x\right)$ satisfy the same structure conditions as the functions $p_{k}, \lambda_{k}^{*}$ and $f_{k}$ that are independent of $k$ in the contradiction argument.

Now, in order to improve the flatness in some possibly new direction we perform a non-homogeneous blow up.

Lemma 3.4. Let $u_{k} \in F\left(\sigma_{k}, \sigma_{k} ; \tau_{k}\right)$ in $B_{1}$ with power $p_{k}$, slope $\lambda_{k}^{*}$ and rhs $f_{k}$ such that $1<p_{\min } \leq$ $p_{k}(x) \leq p_{\max }<\infty, 0<\lambda_{\min } \leq \lambda_{k}^{*}(x) \leq \lambda_{\max }<\infty,\left|\nabla p_{k}\right| \leq L_{1} \rho_{k},\left|f_{k}\right| \leq L_{2} \rho_{k},\left[\lambda_{k}^{*}\right]_{C^{\alpha^{*}}} \leq C^{*} \rho_{k}^{\alpha^{*}}$ with $C^{*} \rho_{k}^{\alpha^{*}} \leq \lambda_{k}^{*}(0) \tau_{k}, \sigma_{k} \rightarrow 0$ and $\frac{\tau_{k}}{\sigma_{k}^{2}} \rightarrow 0$ as $k \rightarrow \infty$.

For $y \in B_{1}^{\prime}$, let

$$
\begin{aligned}
& F_{k}^{+}(y):=\sup \left\{h /\left(y, \sigma_{k} h\right) \in \partial\left\{u_{k}>0\right\}\right\}, \\
& F_{k}^{-}(y):=\inf \left\{h /\left(y, \sigma_{k} h\right) \in \partial\left\{u_{k}>0\right\}\right\} .
\end{aligned}
$$

Then, for a subsequence,

(1) $F(y):=\limsup _{\substack{z \rightarrow y \\ k \rightarrow \infty}} F_{k}^{+}(z)=\liminf _{\substack{z \rightarrow y \\ k \rightarrow \infty}} F_{k}^{-}(z)$ for every $y \in B_{1}^{\prime}$.

Moreover, $F_{k}^{+} \rightarrow F, F_{k}^{-} \rightarrow F$ uniformly, $F$ is continuous, $F(0)=0$ and $|F| \leq 1$.

(2) $F$ is subharmonic.

Proof. (1) is proved exactly as in Lemma 7.3 in [1].

In order to prove (2), we take $g$ a harmonic function in a neighborhood of $B_{r}^{\prime}\left(y_{0}\right) \subset \subset B_{1}^{\prime}$ with $g>F$ on $\partial B_{r}^{\prime}\left(y_{0}\right)$ and $g\left(y_{0}\right)<F\left(y_{0}\right)$ and get a contradiction. We define the sets $Z_{+}(\phi), Z_{-}(\phi)$ and $Z_{0}(\phi)$ as in the previous papers. That is,

$$
Z:=B_{r}^{\prime}\left(y_{0}\right) \times \mathbb{R}, \quad Z_{+}(\phi):=\{(y, h) \in Z / h>\phi(y)\}
$$

and corresponding definitions for $Z_{-}(\phi), Z_{0}(\phi)$.

Observe that we may assume that $\mathcal{H}^{N-1}\left(Z_{0}\left(\sigma_{k} g\right) \cap \partial\left\{u_{k}>0\right\}\right)=0$. If not, we replace $g$ by $g+c_{0}$ for some small enough constant $c_{0}$. 
In fact, let $c_{1}>0$ small such that $g\left(y_{0}\right)<g\left(y_{0}\right)+c<F\left(y_{0}\right)$ for $0<c<c_{1}$. Since by Theorem $2.1 \mathcal{H}^{N-1}\left(D \cap \partial\left\{u_{k}>0\right\}\right)<\infty$ for every $D \subset \subset B_{1}$, we see that

$$
\left|\left\{(y, h) \in Z / \sigma_{k} g(y)<h<\sigma_{k}\left(g(y)+c_{1}\right)\right\} \cap \partial\left\{u_{k}>0\right\}\right|=0,
$$

which implies that $\int_{0}^{c_{1}} H_{k}(c) d c=0$, for $H_{k}(c)=\mathcal{H}^{N-1}\left(Z_{0}\left(\sigma_{k}(g+c)\right) \cap \partial\left\{u_{k}>0\right\}\right)$. Then, we can take $c_{0} \in\left(0, c_{1}\right)$ such that $H_{k}\left(c_{0}\right)=0$ for every $k$, and now replacing $g$ by $g+c_{0}$ we have $\mathcal{H}^{N-1}\left(Z_{0}\left(\sigma_{k} g\right) \cap \partial\left\{u_{k}>0\right\}\right)=0$.

In the following we denote $Z_{+}=Z_{+}\left(\sigma_{k} g\right)$ and similarly $Z_{-}$and $Z_{0}$.

Now, by using the representation formula (Theorem 2.1) and proceeding as in [1], Lemma 7.5, we get

$$
\int_{\left\{u_{k}>0\right\} \cap Z_{0}}\left|\nabla u_{k}\right|^{p_{k}(x)-2} \nabla u_{k} \cdot \nu d \mathcal{H}^{N-1}=\int_{\partial\left\{u_{k}>0\right\} \cap Z_{+}} q_{u_{k}} d \mathcal{H}^{N-1}+\int_{\left\{u_{k}>0\right\} \cap Z_{+}} f_{k} d x .
$$

Since $q_{u_{k}} \geq 0$ and $q_{u_{k}}(x)=\lambda_{k}^{*}(x)^{p_{k}(x)-1} \quad \mathcal{H}^{N-1}-$ a.e. on $\partial_{\text {red }}\left\{u_{k}>0\right\}$,

$$
\begin{aligned}
& \int_{\partial\left\{u_{k}>0\right\} \cap Z_{+}} q_{u_{k}} d \mathcal{H}^{N-1} \geq \int_{\partial_{\text {red }}\left\{u_{k}>0\right\} \cap Z_{+}} \lambda_{k}^{* p_{k}-1} d \mathcal{H}^{N-1} \\
& \quad \geq \min \left\{\left(\lambda_{k}^{*}(0)\left(1-C^{* *} \rho_{k}^{\alpha^{*}}\right)\right)^{p_{k}^{+}-1},\left(\lambda_{k}^{*}(0)\left(1-C^{* *} \rho_{k}^{\alpha^{*}}\right)\right)^{p_{k}^{-}-1}\right\} \mathcal{H}^{N-1}\left(\partial_{\text {red }}\left\{u_{k}>0\right\} \cap Z_{+}\right)
\end{aligned}
$$

where $C^{* *}=\frac{C^{*}}{\lambda_{\min }}, p_{k}^{+}=\sup _{B_{1}} p_{k}$ and $p_{k}^{-}=\inf _{B_{1}} p_{k}$. Recall that $p_{k}^{+}-p_{k}^{-} \leq L_{1} \rho_{k}$.

On the other hand,

$$
\int_{\left\{u_{k}>0\right\} \cap Z_{+}} f_{k} d x \geq-L_{2} \rho_{k}\left|\left\{u_{k}>0\right\} \cap Z_{+}\right| .
$$

Finally,

$$
\begin{aligned}
& \int_{\left\{u_{k}>0\right\} \cap Z_{0}}\left|\nabla u_{k}\right|^{p_{k}(x)-2} \nabla u_{k} \cdot \nu d \mathcal{H}^{N-1} \\
& \quad \leq \max \left\{\left(\lambda_{k}^{*}(0)\left(1+\tau_{k}\right)\right)^{p_{k}^{+}-1},\left(\lambda_{k}^{*}(0)\left(1+\tau_{k}\right)\right)^{p_{k}^{-}-1}\right\} \mathcal{H}^{N-1}\left(\left\{u_{k}>0\right\} \cap Z_{0}\right) .
\end{aligned}
$$

From now on, in order to simplify the computations, we assume that $\lambda_{k}^{*}(0) \geq 1$. The final result will be the same if not.

By (3.10), (3.11) and (3.12),

$$
\begin{aligned}
& \lambda_{k}^{*}(0)^{p_{k}^{-}-1}\left(1-C^{* *} \rho_{k}^{\alpha^{*}}\right)^{p_{k}^{+}-1} \mathcal{H}^{N-1}\left(\partial_{\mathrm{red}}\left\{u_{k}>0\right\} \cap Z_{+}\right) \\
& \quad \leq L_{2} \rho_{k}\left|\left\{u_{k}>0\right\} \cap Z_{+}\right|+\lambda_{k}^{*}(0)^{p_{k}^{+}-1}\left(1+\tau_{k}\right)^{p_{k}^{+}-1} \mathcal{H}^{N-1}\left(\left\{u_{k}>0\right\} \cap Z_{0}\right) .
\end{aligned}
$$

Therefore,

$$
\begin{aligned}
\mathcal{H}^{N-1} & \left(\partial_{\text {red }}\left\{u_{k}>0\right\} \cap Z_{+}\right) \\
& \leq \lambda_{k}^{*}(0)^{p_{k}^{+}-p_{k}^{-}}\left(\frac{1+\tau_{k}}{1-C^{* *} \rho_{k}^{\alpha^{*}}}\right)^{p_{k}^{+}-1} \mathcal{H}^{N-1}\left(\left\{u_{k}>0\right\} \cap Z_{0}\right) \\
& +\frac{L_{2} \rho_{k}}{\lambda_{k}^{*}(0)^{p_{k}^{-}-1}\left(1-C^{* *} \rho_{k}^{\alpha^{*}}\right)^{p_{k}^{+}-1}}\left|\left\{u_{k}>0\right\} \cap Z_{+}\right| .
\end{aligned}
$$

Now, we use the excess area formula Lemma 7.5 in [1] (with $E_{k}=\left\{u_{k}>0\right\} \cup Z_{-}$) that states that, since $F\left(y_{0}\right)>g\left(y_{0}\right)$,

$$
\mathcal{H}^{N-1}\left(\partial_{\text {red }} E_{k} \cap Z\right) \geq \mathcal{H}^{N-1}\left(Z_{0}\right)+c \sigma_{k}^{2}
$$


for $k$ large.

Therefore, since there holds $Z \cap \partial E_{k}=\left(Z_{+} \cap \partial\left\{u_{k}>0\right\}\right) \cup\left(Z_{0} \cap\left\{u_{k}=0\right\}\right)$ and (3.14), we obtain

$$
\begin{aligned}
\mathcal{H}^{N-1}\left(Z_{+} \cap \partial_{\text {red }}\left\{u_{k}>0\right\}\right) & \geq \mathcal{H}^{N-1}\left(Z \cap \partial_{\text {red }} E_{k}\right)-\mathcal{H}^{N-1}\left(Z_{0} \cap\left\{u_{k}=0\right\}\right) \\
& \geq \mathcal{H}^{N-1}\left(Z_{0}\right)+c \sigma_{k}^{2}-\mathcal{H}^{N-1}\left(Z_{0} \cap\left\{u_{k}=0\right\}\right) \\
& =\mathcal{H}^{N-1}\left(Z_{0} \cap\left\{u_{k}>0\right\}\right)+c \sigma_{k}^{2} .
\end{aligned}
$$

From here, using the facts that

$$
\lambda_{k}^{*}(0)^{p_{k}^{+}-p_{k}^{-}}\left(\frac{1+\tau_{k}}{1-C^{* *} \rho_{k}^{\alpha^{*}}}\right)^{p_{k}^{+}-1}-1 \leq C_{0}\left(\tau_{k}+\rho_{k}^{\alpha^{*}}\right)
$$

and

$$
\frac{L_{2} \rho_{k}}{\lambda_{k}^{*}(0)^{p_{k}^{-}-1}\left(1-C^{* *} \rho_{k}^{\alpha^{*}}\right)^{p_{k}^{+}-1}} \leq C_{1} \rho_{k},
$$

together with $\left|\left\{u_{k}>0\right\} \cap Z_{+}\right| \leq\left|B_{1}\right| \leq C, \mathcal{H}^{N-1}\left(\left\{u_{k}>0\right\} \cap Z_{0}\right) \leq \mathcal{H}^{N-1}\left(Z_{0}\right) \leq C$, (3.13) and (3.15), we get

$$
c \sigma_{k}^{2} \leq C C_{0}\left(\tau_{k}+\rho_{k}^{\alpha^{*}}\right)+C C_{1} \rho_{k} \leq C_{2}\left(\tau_{k}+\rho_{k}^{\alpha^{*}}\right) .
$$

This is a contradiction to our assumptions that $C^{*} \rho_{k}^{\alpha^{*}} \leq \lambda_{k}^{*}(0) \tau_{k}$ and $\frac{\tau_{k}}{\sigma_{k}^{2}} \rightarrow 0$.

The following lemma was proved in [2] with $c=1$. The result is obtained by rescaling the $h$ variable.

Lemma 3.5. Let $w(y, h)$ be such that

(a) $\sum_{i=1}^{N-1} w_{y_{i} y_{i}}+c w_{h h}=0$ in $B_{1} \cap\{h<0\}$ with $c>0$.

(b) $w(y, h) \rightarrow g$ in $L^{1}$ as $h \nearrow 0$.

(c) $g$ is subharmonic and continuous in $B_{1}^{\prime}, g(0)=0$.

(d) $w(0, h) \leq C|h|$.

(e) $w \geq-C$.

Then, there exists $C_{0}$ depending only on $C, N$ and $c$ such that, for every $y \in B_{1 / 2}^{\prime}$,

$$
\int_{0}^{1 / 2} \frac{1}{r^{2}}\left(f_{\partial B_{r}^{\prime}(y)} g(z) d \mathcal{H}^{N-2}\right) d r \leq C_{0} .
$$

Then, we have

Lemma 3.6. Let $u_{k}, p_{k}, \lambda_{k}^{*}, f_{k}, \rho_{k}, \sigma_{k}$ as in Lemma 3.4. Let $F_{k}^{+}, F_{k}^{-}$and $F$ as in that lemma. There exists $C=C\left(N, p_{\min }, p_{\max }, \lambda_{\min }, \lambda_{\max }\right)$ such that, if $y_{0} \in B_{1 / 2}^{\prime}$,

$$
\int_{0}^{1 / 4} \frac{1}{r^{2}}\left(f_{\partial B_{r}^{\prime}\left(y_{0}\right)}\left(F-F\left(y_{0}\right)\right) d \mathcal{H}^{N-2}\right) d r \leq C .
$$

Proof. The proof follows the lines of the previously cited papers. The idea is that the function $2\left(F\left(y_{0}+\frac{1}{2} y\right)-F\left(y_{0}\right)\right)$ will take the place of the function $g$ in Lemma 3.5.

We write down the proof for the reader's convenience since we cannot assume that $\lambda_{k}^{*}(0)=1$ and we have a right hand side in the equation that was not present in the previous papers. We let $y_{0} \in B_{1 / 2}^{\prime}$ and consider the functions $\bar{u}_{k}(y, h)=2 u_{k}\left(y_{0}+\frac{1}{2} y, \sigma_{k} F_{k}^{+}\left(y_{0}\right)+\frac{1}{2} h\right)$ in $B_{1}$. From the fact that $u_{k} \in F\left(\sigma_{k}, \sigma_{k} ; \tau_{k}\right)$ in $B_{1}$ we deduce that $\bar{u}_{k} \in F\left(4 \sigma_{k}, 4 \sigma_{k} ; \tau_{k}\right)$ in $B_{1}$.

In fact, we denote $\left(x^{\prime}, x_{N}\right)=\left(y_{0}+\frac{1}{2} y, \sigma_{k} F_{k}^{+}\left(y_{0}\right)+\frac{1}{2} h\right)$ and recall that $\left|F_{k}^{+}\right| \leq 1$. Then we have for $y \in B_{1}^{\prime}, h>4 \sigma_{k}$ that $x_{N}>\sigma_{k} F_{k}^{+}\left(y_{0}\right)+2 \sigma_{k} \geq \sigma_{k}$ implying that $\bar{u}_{k}(y, h)=0$. 
On the other hand, for $y \in B_{1}^{\prime}, h<-4 \sigma_{k}$ we have $x_{N}<\sigma_{k} F_{k}^{+}\left(y_{0}\right)-2 \sigma_{k} \leq-\sigma_{k}$. This implies that $\bar{u}_{k}(y, h)=2 u_{k}\left(x^{\prime}, x_{N}\right) \geq-2 \lambda_{k}^{*}(0)\left[x_{N}+\sigma_{k}\right] \geq-\lambda_{k}^{*}(0)\left[h+4 \sigma_{k}\right]$.

Finally, we see that $\left|\nabla \bar{u}_{k}(y, h)\right|=\left|\nabla u_{k}\left(y_{0}+\frac{1}{2} y, \sigma_{k} F_{k}^{+}\left(y_{0}\right)+\frac{1}{2} h\right)\right| \leq \lambda_{k}^{*}(0)\left(1+\tau_{k}\right)$ and we conclude that $\bar{u}_{k} \in F\left(4 \sigma_{k}, 4 \sigma_{k} ; \tau_{k}\right)$ in $B_{1}$.

Observe that by this change of variables the function $F_{k}^{+}(y)$ has been replaced by $2\left(F_{k}^{+}\left(y_{0}+\right.\right.$ $\left.\left.\frac{1}{2} y\right)-F_{k}^{+}\left(y_{0}\right)\right)$.

Thus, from now on we may assume that $u_{k} \in F\left(4 \sigma_{k}, 4 \sigma_{k} ; \tau_{k}\right)$ in $B_{1}$ and $y_{0}=0$. Let

$$
w_{k}(y, h)=\frac{u_{k}(y, h)+\lambda_{k}^{*}(0) h}{\sigma_{k}} .
$$

Then, given $0<\delta<\frac{1}{2}$, we take $k \geq k_{\delta}$ so that $\lambda_{k}^{*}(0) / 2 \leq\left|\nabla u_{k}\right| \leq 2 \lambda_{k}^{*}(0)$ in $B_{1-\delta} \cap\left\{h \leq-C_{\delta} \sigma_{k}\right\}$ with $C_{\delta}$ the constant in Lemma 3.3 with $R=1-\delta$. We have

$$
\mathcal{T}_{k} w_{k}:=\sum_{i j} b_{i j}^{k}(x) w_{k x_{i} x_{j}}+\sum_{j} b_{j}^{k}(x) w_{k x_{j}}=\frac{b_{N}^{k}}{\sigma_{k}} \lambda_{k}^{*}(0)+\frac{f_{k}}{\sigma_{k}}\left|\nabla u_{k}\right|^{2-p_{k}} \quad \text { in } \quad B_{1-\delta} \cap\left\{h \leq-C_{\delta} \sigma_{k}\right\} .
$$

Here $b_{i j}^{k}(x)=\delta_{i j}+\left(p_{k}(x)-2\right) \frac{u_{k_{x_{i}}} u_{k_{x_{j}}}}{\left|\nabla u_{k}\right|^{2}}$ and $b_{j}^{k}(x)=p_{k_{x_{j}}} \log \left|\nabla u_{k}\right|$. Therefore, $\mathcal{T}_{k}$ is a uniformly elliptic operator with ellipticity and bounds of the coefficients independent of $k$. Namely, they satisfy (3.7) and

(see $(3.8))$.

$$
\left|b^{k}\right| \leq \bar{C}_{0} \rho_{k}
$$

On the other hand, the right hand side satisfies

$$
\frac{b_{N}^{k}}{\sigma_{k}} \lambda_{k}^{*}(0)+\frac{f_{k}}{\sigma_{k}}\left|\nabla u_{k}\right|^{2-p_{k}} \leq K_{0} \frac{\rho_{k}}{\sigma_{k}} \rightarrow 0 \quad \text { as } \quad k \rightarrow \infty .
$$

We will divide the proof into several steps.

(i) We prove that there exists a constant $C>0$ such that $\left\|w_{k}\right\|_{L^{\infty}\left(B_{1}^{-}\right)} \leq C$.

In fact, recall that $u_{k} \in F\left(4 \sigma_{k}, 4 \sigma_{k} ; \tau_{k}\right)$ in $B_{1}$ so $u_{k}(0,0)=0$ and $\left|\nabla u_{k}\right| \leq \lambda_{k}^{*}(0)\left(1+\tau_{k}\right)$. On the other hand, there holds that $u_{k}(y, h)=0$ if $h \geq 4 \sigma_{k}$. Therefore,

$$
u_{k}(y, h) \leq \lambda_{k}^{*}(0)\left(1+\tau_{k}\right)\left(4 \sigma_{k}-h\right)
$$

so that, if $-K \leq h \leq 0$,

$$
w_{k}(y, h) \leq 4 \lambda_{k}^{*}(0)\left(1+\tau_{k}\right)-\lambda_{k}^{*}(0) \frac{\tau_{k}}{\sigma_{k}} h \leq C .
$$

On the other hand, if $h<-4 \sigma_{k}$, since $u_{k} \in F\left(4 \sigma_{k}, 4 \sigma_{k} ; \tau_{k}\right)$ in $B_{1}$, by (2) in Definition 3.1,

$$
w_{k}(y, h)=\frac{u_{k}(y, h)+\lambda_{k}^{*}(0) h}{\sigma_{k}} \geq-\frac{\lambda_{k}^{*}(0)\left(h+4 \sigma_{k}\right)-\lambda_{k}^{*}(0) h}{\sigma_{k}}=-4 \lambda_{k}^{*}(0) .
$$

Finally, if $-4 \sigma_{k} \leq h \leq 0$,

$$
\begin{aligned}
w_{k}(y, h) & \geq-\frac{\lambda_{k}^{*}(0)\left(1+\tau_{k}\right)\left(4 \sigma_{k}-h\right)-\lambda_{k}^{*}(0) h}{\sigma_{k}} \\
& =-4 \lambda_{k}^{*}(0)\left(1+\tau_{k}\right)+\frac{\lambda_{k}^{*}(0)\left(2+\tau_{k}\right) h}{\sigma_{k}} \\
& \geq-C .
\end{aligned}
$$

(ii) Uniform bounds of first and second order derivatives. 
Recall that $w_{k}$ satisfies (3.17) that is uniformly elliptic with ellipticity constants and bounds of the coefficients independent of $k$ in $B_{1-\delta} \cap\left\{h<-C_{\delta} \sigma_{k}\right\}$. By step (i) we then have

$$
\left\|w_{k}\right\|_{C^{1, \alpha}(\mathcal{K})} \leq C_{\mathcal{K}} \quad \forall \mathcal{K} \subset \subset B_{1}^{-} .
$$

and, for every $1<q<\infty$,

$$
\left\|w_{k}\right\|_{W^{2, q}(\mathcal{K})} \leq C_{\mathcal{K}} \quad \forall \mathcal{K} \subset \subset B_{1}^{-} .
$$

Hence, for a subsequence that we still call $w_{k}$, there exists $w \in C^{1, \alpha} \cap W^{2, q}$ such that $w_{k} \rightarrow w$ in $C^{1}(\mathcal{K})$ and weakly in $W^{2, q}(\mathcal{K})$ for every $\mathcal{K} \subset \subset B_{1}^{-}$.

(iii) Determining the equation satisfied by $w$.

Let $c_{i j}=\delta_{i j}+\left(p_{0}-2\right) \delta_{i N} \delta_{j N}$ where $p_{\min } \leq p_{0} \leq p_{\max }$ is the uniform limit of the sequence of functions $p_{k}$ (for a subsequence). Then, $b_{i j}^{k} \rightarrow c_{i j}$ uniformly on compact subsets of $B_{1}^{-}$. In fact, by the uniform estimates of the gradient of $w_{k}$ we have that

$$
\left|\nabla u_{k}(y, h)+\lambda_{k}^{*}(0) e_{N}\right|=\left|\nabla\left(u_{k}(y, h)+\lambda_{k}^{*}(0) h\right)\right| \leq C_{\mathcal{K}} \sigma_{k}
$$

if $k \geq k_{\mathcal{K}}$ and $\mathcal{K} \subset \subset B_{1}^{-}$.

Let $\lambda_{0}^{*}=\lim _{k \rightarrow \infty} \lambda_{k}^{*}(0)$ (for a subsequence). Then, by (3.21) $\nabla u_{k} \rightarrow-\lambda_{0}^{*} e_{N}$ uniformly on compact subsets of $B_{1}^{-}$. Since $\lambda_{0}^{*} \geq \lambda_{\min }>0$, there holds that

$$
\frac{u_{k x_{i}} u_{k x_{j}}}{\left|\nabla u_{k}\right|^{2}} \rightarrow \delta_{i N} \delta_{j N}
$$

uniformly on compact subsets of $B_{1}^{-}$. And we have proved the convergence.

On the other hand, $\left|b_{j}^{k}(x)\right| \leq C_{0} \sigma_{k}$. Therefore, by passing to the limit in (3.17) we get

$$
\sum_{i j} c_{i j} w_{x_{i} x_{j}}=0 \quad \text { in } B_{1}^{-} .
$$

(iv) Bounds of $w$.

Recalling that $\left|\nabla u_{k}\right| \leq \lambda_{k}^{*}(0)\left(1+\tau_{k}\right)$, we get

$$
\frac{\partial}{\partial h} w_{k}(y, h) \geq-\frac{\lambda_{k}^{*}(0)\left(1+\tau_{k}\right)-\lambda_{k}^{*}(0)}{\sigma_{k}}=-\lambda_{k}^{*}(0) \frac{\tau_{k}}{\sigma_{k}} .
$$

Thus, for $h<0$,

$$
w_{k}(0, h) \leq \lambda_{k}^{*}(0) \frac{\tau_{k}}{\sigma_{k}}|h| \rightarrow 0 \quad \text { as } \quad k \rightarrow \infty .
$$

Passing to the limit, we find that

$$
w(0, h) \leq 0 \quad \text { for } \quad h<0 .
$$

(v) Let us see that $w(y, h) \rightarrow \lambda_{0}^{*} F(y)$ as $h \rightarrow 0^{-}$, uniformly in $B_{1-\delta}^{\prime}$ for every $0<\delta<1$. First, as in $[2,8]$, we can prove that

$$
w_{k}\left(y, \sigma_{k} h\right)-\lambda_{0}^{*} F(y) \rightarrow 0 \quad \text { uniformly in } \quad B_{1-\delta}^{\prime} \times\left[-K,-2 C_{\delta}\right]
$$

for every $K>2 C_{\delta}$ and every $0<\delta<1$. We omit this proof, that relies heavily on Proposition 3.1 (see [2] for the proof).

In order to get the result, following the ideas in $[2,8]$, we construct a barrier. First, for $\delta>0$ we let $\Omega_{\delta}$ a smooth domain such that

$$
B_{1-2 \delta}^{-} \subset \Omega_{\delta} \subset B_{1-\delta}^{-} .
$$


For $\varepsilon>0$ small, we let $g_{\varepsilon} \in C^{3}\left(\partial \Omega_{\delta}\right)$ such that $\left\|g_{\varepsilon}\right\|_{C^{3}\left(\partial \Omega_{\delta}\right)} \leq C$ with $C$ independent of $\varepsilon$ and $\delta$ and

$$
\begin{aligned}
\lambda_{0}^{*} F-2 \varepsilon \leq & g_{\varepsilon} \leq \lambda_{0}^{*} F-\varepsilon & \text { in } \partial \Omega_{\delta} \cap \partial B_{1-3 \delta}^{-} \cap\{h=0\} \\
& g_{\varepsilon} \leq \lambda_{0}^{*} F-\varepsilon & \text { in } \partial \Omega_{\delta} \cap\{h=0\} \\
& g_{\varepsilon} \leq w-\varepsilon & \text { in } \partial \Omega_{\delta} \cap\{h<0\} .
\end{aligned}
$$

Then, we let $\phi_{\varepsilon}$ the solution to

$$
\left\{\begin{array}{lll}
\sum_{i j} c_{i j} \phi_{\varepsilon_{x_{i} x_{j}}}=1 & \text { in } & \Omega_{\delta} \\
\phi_{\varepsilon}=g_{\varepsilon} & \text { on } & \partial \Omega_{\delta}
\end{array}\right.
$$

with $c_{i j}$ as in (3.22).

On one hand, if $k \geq k(\varepsilon, \delta)$,

$$
\phi_{\varepsilon} \leq w_{k} \quad \text { on } \quad \partial \Omega_{\delta} \cap\left\{h<-2 C_{\delta} \sigma_{k}\right\} .
$$

On the other hand, since $\left\|\phi_{\varepsilon}\right\|_{C^{2}\left(\overline{\Omega_{\delta}}\right)} \leq C$, there holds that, for $K>2 C_{\delta}$ and $k \geq k(\varepsilon, \delta, K)$,

$$
\phi_{\varepsilon} \leq w_{k} \quad \text { on } \quad \Omega_{\delta} \cap\left\{h=-K \sigma_{k}\right\} .
$$

Recall that, by Lemma 3.3, we have

$$
\left|\nabla u_{k}\right| \geq \frac{\lambda_{k}^{*}(0)}{2} \quad \text { in } \quad B_{1-\delta} \cap\left\{h<-C_{\delta} \sigma_{k}\right\}
$$

and there holds (3.17) and (3.18). Therefore,

$$
\mathcal{T}_{k} w_{k} \leq K_{0} \frac{\rho_{k}}{\sigma_{k}} \leq \frac{1}{2} \quad \text { in } \quad \Omega_{\delta} \cap\left\{h<-K \sigma_{k}\right\}
$$

if $k \geq k_{0}$.

Let us see that

$$
\mathcal{T}_{k} \phi_{\varepsilon} \geq \frac{1}{2} \quad \text { in } \quad \Omega_{\delta} \cap\left\{h<-K \sigma_{k}\right\}
$$

if $K$ is large independently of $\varepsilon$ and $k$ is large independently of $\varepsilon$ and $K$. In fact, for $x \in \Omega_{\delta}$,

$$
\begin{aligned}
\mathcal{T}_{k} \phi_{\varepsilon} & =\sum_{i j} c_{i j} \phi_{\varepsilon x_{i} x_{j}}+\sum_{i j}\left(b_{i j}^{k}(x)-c_{i j}\right) \phi_{\varepsilon x_{i} x_{j}}+\sum_{j} b_{j}^{k}(x) \phi_{\varepsilon x_{j}} \\
& \geq 1-\left\|D^{2} \phi_{\varepsilon}\right\|_{L^{\infty}} \sum_{i j}\left\|b_{i j}^{k}-c_{i j}\right\|_{L^{\infty}}-\left\|b^{k}\right\|_{L^{\infty}}\left\|\nabla \phi_{\varepsilon}\right\|_{L^{\infty}} .
\end{aligned}
$$

On one hand, $\left\|b^{k}\right\|_{L^{\infty}} \leq C_{0} \sigma_{k} \rightarrow 0$ as $k \rightarrow \infty$. On the other hand, by elliptic estimates up to the boundary $\left\{h=-K \sigma_{k}\right\}$, since we have proved that $\left|w_{k}\right| \leq C$,

$$
\begin{aligned}
& \left\|\nabla\left(u_{k}+\lambda_{k}^{*}(0) h\right)\right\|_{L^{\infty}\left(\left\{h \leq-K \sigma_{k}\right\}\right)}=\sigma_{k}\left\|\nabla w_{k}\right\|_{L^{\infty}\left(\left\{h \leq-K \sigma_{k}\right\}\right)} \\
& \quad \leq \sigma_{k} C \frac{\rho_{k} / \sigma_{k}+1}{\left(K-C_{\delta}\right) \sigma_{k}} \leq \frac{2 C}{K-C_{\delta}} \quad \text { in } \quad \Omega_{\delta} \cap\left\{h<-K \sigma_{k}\right\} .
\end{aligned}
$$

Then, as $\frac{\lambda_{k}^{*}(0)}{2} \leq\left|\nabla u_{k}\right| \leq 2 \lambda_{k}^{*}(0)$ in that set and $p_{k}(x)-p_{0} \rightarrow 0$ uniformly in $B_{1}$,

$$
\left\|b_{i j}^{k}-c_{i j}\right\|_{L^{\infty}\left(B_{1} \cap\left\{h \leq-K \sigma_{k}\right\}\right)} \leq \frac{C}{K-C_{\delta}}+o_{k}(1) .
$$

We conclude, by taking $K$ large enough independent of $k$ and $\varepsilon$ and then, $k$ large, that (3.26) holds. 
Therefore, $\phi_{\varepsilon} \leq w_{k}$ in $\Omega_{\delta} \cap\left\{h \leq-K \sigma_{k}\right\}$. By letting $k \rightarrow \infty$ we find that $\phi_{\varepsilon} \leq w$ in $\Omega_{\delta} \cap\{h<0\}$ and then, by letting $h \rightarrow 0^{-}$,

$$
\liminf _{h \rightarrow 0^{-}} w(y, h) \geq \lim _{h \rightarrow 0^{-}} \phi_{\varepsilon}(y, h) \geq \lambda_{0}^{*} F(y)-2 \varepsilon \quad \text { for } \quad y \in B_{1-3 \delta}^{\prime} .
$$

In order to get a bound from above, we recall (3.23) and get,

$$
w_{k}(y, h)-w_{k}\left(y,-K \sigma_{k}\right) \leq-C \frac{\tau_{k}}{\sigma_{k}}|h| \quad \text { if } \quad h \leq-K \sigma_{k} .
$$

On the other hand, $w_{k}\left(y,-K \sigma_{k}\right) \rightarrow \lambda_{0}^{*} F(y)$ uniformly in $B_{1-\delta}^{\prime}$. Hence, if $k$ is large, and $(y, h) \in$ $B_{1-\delta}^{-} \cap\left\{h \leq-K \sigma_{k}\right\}$,

$$
w_{k}(y, h) \leq \lambda_{0}^{*} F(y)+2 \varepsilon
$$

and we deduce that, for $(y, h) \in B_{1-\delta}^{-}$,

$$
w(y, h) \leq \lambda_{0}^{*} F(y)+2 \varepsilon .
$$

Therefore,

$$
\limsup _{h \rightarrow 0^{-}} w(y, h) \leq \lambda_{0}^{*} F(y)+2 \varepsilon \quad \text { uniformly in } \quad B_{1-\delta}^{\prime} .
$$

Since $\varepsilon$ is arbitrary, we conclude that, for every $0<\delta<1$,

$$
\lim _{h \rightarrow 0^{-}} w(y, h)=\lambda_{0}^{*} F(y) \quad \text { uniformly for } y \in B_{1-3 \delta}^{\prime} .
$$

(vi) Final step.

We apply Lemma 3.5 to the function $w$ and recall that when writing $w(y, 0)$ in the original variables we get $2\left(F\left(y_{0}+\frac{1}{2} y\right)-F\left(y_{0}\right)\right)$. So, the result is proved.

Corollary 3.1. Let $u_{k}, p_{k}, \lambda_{k}^{*}, f_{k}, \rho_{k}, \sigma_{k}$ and $F$ as in Lemma 3.4. There exists a constant $C=$ $C\left(N, p_{\min }, p_{\max }, \lambda_{\min }, \lambda_{\max }\right)$ and, for every $0<\theta<1$ there exist $c_{\theta}=c_{\theta}\left(N, p_{\min }, p_{\max }, \lambda_{\min }, \lambda_{\max }, \theta\right)$, a ball $B_{r}^{\prime}$ and $\ell \in \mathbb{R}^{N-1}$ such that

$$
c_{\theta} \leq r \leq \theta, \quad|\ell| \leq C, \quad F(y) \leq \ell \cdot y+\frac{\theta}{2} r \quad \text { for }|y| \leq r .
$$

Proof. The result is a consequence of Lemma 3.6 and the proof follows as Lemmas 7.7 and 7.8 in [1].

Now, we apply the corollary to a weak flat solution $u$ if $\sigma$ is small enough.

Lemma 3.7. Let $p \in \operatorname{Lip}\left(B_{\rho}\right), \lambda^{*} \in C^{\alpha^{*}}\left(B_{\rho}\right), f \in L^{\infty}\left(B_{\rho}\right)$ such that $1<p_{\min } \leq p(x) \leq p_{\max }<\infty$, $0<\lambda_{\min } \leq \lambda^{*}(x) \leq \lambda_{\max }<\infty$ with $|\nabla p| \leq L_{1},|f| \leq L_{2}$ and $\left[\lambda^{*}\right]_{C^{*}\left(B_{\rho}\right)} \leq C^{*}$. Let $0<\theta<1$. There exists $\sigma_{\theta}=\sigma_{\theta}\left(\theta, N, p_{\min }, p_{\max }, \lambda_{\min }, \lambda_{\max }, L_{1}, L_{2}, C^{*}\right)$ such that, if

$$
u \in F(\sigma, \sigma ; \tau) \text { in } B_{\rho} \text { in direction } \nu
$$

with power $p$, slope $\lambda^{*}$ and rhs $f$ and, if $C^{*} \rho^{\alpha^{*}} \leq \lambda^{*}(0) \tau, \sigma \leq \sigma_{\theta}$ and $\tau \leq \sigma_{\theta} \sigma^{2}$ there holds that $u \in F(\theta \sigma, 1 ; \tau)$ in $B_{\bar{\rho}}$ in direction $\bar{\nu}$

with the same power, slope and rhs and

$$
c_{\theta} \rho \leq \bar{\rho} \leq \theta \rho, \quad|\nu-\bar{\nu}| \leq C \sigma .
$$

Here $c_{\theta}$ and $C$ are the constants in Corollary 3.1.

Proof. It follows as Lemma 7.9 in [1] by applying Corollary 3.1 to $\bar{u}_{k}(x)=\frac{1}{\rho_{k}} u_{k}\left(\rho_{k} x\right)$. 
Now, in order to improve on the gradient in the flatness class, we find an equation to which $v=|\nabla u|$ is a subsolution.

Lemma 3.8. Let $p \in W^{1, \infty}(\Omega) \cap W^{2, q}(\Omega)$ with $1<p_{\min } \leq p(x) \leq p_{\max }<\infty$ in $\Omega$ and $f \in$ $L^{\infty}(\Omega) \cap W^{1, q}(\Omega)$ for some $q \geq 1$.

Let $u$ such that $\Delta_{p(x)} u=f$ and $0<c \leq|\nabla u| \leq C$ in $\Omega$. There exist $D=\left\{D_{i j}\right\}, B=\left\{b_{j}\right\}$ and $G$ such that

$$
\begin{aligned}
& \bar{\beta}|\xi|^{2} \leq \sum_{i j} D_{i j}(x) \xi_{i} \xi_{j} \leq \bar{\beta}^{-1}|\xi|^{2} \quad \text { for every } \xi \in \mathbb{R}^{N}, x \in \Omega, \\
& \|B\|_{L^{\infty}(\Omega)} \leq \bar{C} \quad, \quad\|G\|_{L^{q}(\Omega)} \leq \bar{C}
\end{aligned}
$$

with $\bar{\beta}=\bar{\beta}\left(p_{\min }, p_{\max }, c, C\right)>0, \bar{C}=\bar{C}\left(p_{\min }, p_{\max }, c, C,\|f\|_{L^{\infty}(\Omega) \cap W^{1, q}(\Omega)},\|p\|_{W^{1, \infty}(\Omega) \cap W^{2, q}(\Omega)}\right)$ such that $v=|\nabla u|$ satisfies

$$
\operatorname{div} D \nabla v+B \cdot \nabla v \geq G
$$

weakly in $\Omega$.

Proof. We start with some notation. For $x \in \Omega, \xi \in \mathbb{R}^{N}$, we let $A(x, \xi)=|\xi|^{p(x)-2} \xi$. First we observe that, by the arguments in Theorem 3.2 in [7], $u \in W_{\text {loc }}^{2,2}(\Omega)$ and then, by using the nondivergence form of the equation, we deduce that $u \in W_{\text {loc }}^{2, t}(\Omega)$ for every $1 \leq t<\infty$ (see Lemma 9.16 in [17]).

Then, taking $\eta \in C_{0}^{\infty}(\Omega)$, letting $\eta_{x_{k}}$ as test function and integrating by parts, we get

$$
\int f \eta_{x_{k}}=\int \frac{\partial A}{\partial x_{k}}(x, \nabla u) \nabla \eta+\sum_{i j} \int a_{i j}(x, \nabla u) u_{x_{j} x_{k}} \eta_{x_{i}}
$$

where $a_{i j}(x, \xi)=\frac{\partial A_{i}}{\partial \xi_{j}}(x, \xi)$.

Observe that (3.28) actually holds for any $\eta \in W_{0}^{1, p(x)}(\Omega)$.

Then, we take $\eta=u_{x_{k}} \psi$ with $0 \leq \psi \in C_{0}^{\infty}(\Omega)$ arbitrary. Hence, by using the ellipticity of $a_{i j}$ and after summation on $k$, we get

$$
\begin{aligned}
\int f \Delta u \psi+\int f\langle\nabla u, \nabla \psi\rangle & \geq \sum_{i, k} \int \frac{\partial A_{i}}{\partial x_{k}}(x, \nabla u) u_{x_{i} x_{k}} \psi \\
& +\sum_{i, k} \int \frac{\partial A_{i}}{\partial x_{k}}(x, \nabla u) u_{x_{k}} \psi_{x_{i}}+\sum_{i, j} \int a_{i j} \sum_{k} u_{x_{k}} u_{x_{j} x_{k}} \psi_{x_{i}} .
\end{aligned}
$$

Now, we denote $D=\left(D_{i j}\right)$ with $D_{i j}=|\nabla u| a_{i j}$, we use that $v_{x_{j}}=\sum_{k} \frac{u_{x_{k} x_{j}} u_{x_{k}}}{|\nabla u|}$ and we integrate by parts the second terms on the left and right hand sides. In fact, since

$$
\frac{\partial A_{i}}{\partial x_{k}}(x, \nabla u)=|\nabla u|^{p(x)-2} \log |\nabla u| u_{x_{i}} p_{x_{k}},
$$

we get

$$
\begin{aligned}
\frac{d}{d x_{i}}\left[\frac{\partial A_{i}}{\partial x_{k}}(x, \nabla u)\right] & =|\nabla u|^{p(x)-2}(\log |\nabla u|)^{2} u_{x_{i}} p_{x_{k}} p_{x_{i}} \\
& +|\nabla u|^{p(x)-2} \log |\nabla u| u_{x_{i}} p_{x_{k} x_{i}}+|\nabla u|^{p(x)-2} \log |\nabla u| u_{x_{i} x_{i}} p_{x_{k}} \\
& +(p(x)-2)|\nabla u|^{p(x)-3} \log |\nabla u| u_{x_{i}} p_{x_{k}} v_{x_{i}}+|\nabla u|^{p(x)-3} u_{x_{i}} p_{x_{k}} v_{x_{i}},
\end{aligned}
$$


so we obtain

$$
\begin{aligned}
-\int\langle\nabla f, \nabla u\rangle \psi & \geq \int\langle D \nabla v, \nabla \psi\rangle+\sum_{i, k} \int \frac{\partial A_{i}}{\partial x_{k}}(x, \nabla u) u_{x_{i} x_{k}} \psi \\
& -\sum_{i, k} \int \frac{d}{d x_{i}}\left[\frac{\partial A_{i}}{\partial x_{k}}(x, \nabla u)\right] u_{x_{k}} \psi-\sum_{i, k} \int \frac{\partial A_{i}}{\partial x_{k}}(x, \nabla u) u_{x_{i} x_{k}} \psi \\
& =\int\langle D \nabla v, \nabla \psi\rangle-\sum_{i, k} \int \frac{d}{d x_{i}}\left[\frac{\partial A_{i}}{\partial x_{k}}(x, \nabla u)\right] u_{x_{k}} \psi
\end{aligned}
$$

Then, by replacing (3.29) in (3.30), it follows

$$
\begin{aligned}
-\int\langle\nabla f, \nabla u\rangle \psi & \geq \int\langle D \nabla v, \nabla \psi\rangle-\int|\nabla u|^{p(x)-2}(\log |\nabla u|)^{2}\langle\nabla u, \nabla p\rangle^{2} \psi \\
& -\int|\nabla u|^{p(x)-2} \log |\nabla u| \sum_{i, k} u_{x_{i}} u_{x_{k}} p_{x_{k} x_{i}} \psi-\int|\nabla u|^{p(x)-2} \log |\nabla u|\langle\nabla u, \nabla p\rangle \Delta u \psi \\
& -\int\left\langle|\nabla u|^{p(x)-3}[(p(x)-2) \log |\nabla u|+1]\langle\nabla u, \nabla p\rangle \nabla u, \nabla v\right\rangle \psi .
\end{aligned}
$$

Finally, since $|\nabla u|^{p(x)-2}\left(\Delta u+(p(x)-2) \sum_{i, j} \frac{u_{x_{i}} u_{x_{j}}}{|\nabla u|^{2}} u_{x_{i} x_{j}}+\log |\nabla u|\langle\nabla u, \nabla p\rangle\right)=f$,

$$
\begin{aligned}
-\int|\nabla u|^{p(x)-2} \log |\nabla u|\langle\nabla u, \nabla p\rangle \Delta u \psi & =-\int f \log |\nabla u|\langle\nabla u, \nabla p\rangle \psi \\
& +\int\left\langle(p(x)-2)|\nabla u|^{p(x)-3} \log |\nabla u|\langle\nabla u, \nabla p\rangle \nabla u, \nabla v\right\rangle \psi \\
& +\int|\nabla u|^{p(x)-2}(\log |\nabla u|)^{2}\langle\nabla u, \nabla p\rangle^{2} \psi .
\end{aligned}
$$

Hence, $v$ satisfies (3.27) with

$$
\begin{aligned}
D_{i j} & =|\nabla u|^{p(x)-1}\left(\delta_{i j}+\frac{(p(x)-2)}{|\nabla u|^{2}} u_{x_{i}} u_{x_{j}}\right), \\
B & =|\nabla u|^{p(x)-3}\langle\nabla u, \nabla p\rangle \nabla u, \\
G & =\langle\nabla f, \nabla u\rangle-f \log |\nabla u|\langle\nabla u, \nabla p\rangle-|\nabla u|^{p(x)-2} \log |\nabla u| \sum_{i, k} u_{x_{i}} u_{x_{k}} p_{x_{k} x_{i}} .
\end{aligned}
$$

Remark 3.1. A similar lemma to Lemma 3.8, valid for the case $f \equiv 0$, was established in reference [6] (Lemma 2.2).

Now, we get an estimate on $|\nabla u|$ close to the free boundary.

Lemma 3.9. Let $p$ and $f$ as in Lemma 3.8 with $q>\max \{1, N / 2\}$ and $\lambda^{*} \in C^{\alpha^{*}}(\Omega)$ with $0<$ $\lambda_{\min } \leq \lambda^{*}(x) \leq \lambda_{\max }<\infty$ in $\Omega$ and $\left[\lambda^{*}\right]_{C^{\alpha^{*}}(\Omega)} \leq C^{*}$. Let $u$ be a weak solution to $P\left(f, p, \lambda^{*}\right)$ in $\Omega$ and let $x_{0} \in \Omega \cap \partial\{u>0\}$ with $B_{4 R}\left(x_{0}\right) \subset \Omega, R \leq 1$. Assume that, for every $r \leq R$,

$$
u \in F(\sigma, 1 ; \infty) \text { in } B_{r}\left(x_{0}\right) \text { in some direction } \nu_{r} \text {, }
$$

with power $p$, slope $\lambda^{*}$ and rhs $f$, with $\sigma \leq 1 / 2$. 
Then, for every $x_{1}$ in $B_{r}\left(x_{0}\right)$,

$$
|\nabla u| \leq \lambda^{*}\left(x_{1}\right)+C\left(\frac{r}{R}\right)^{\gamma} \quad \text { in } \quad B_{r}\left(x_{1}\right) \quad \text { if } \quad r \leq R,
$$

for some constants $C$ and $0<\gamma<1$ depending only on $N, p_{\min }, p_{\max }, \lambda_{\min },\|f\|_{L^{\infty}\left(B_{2 R}\left(x_{0}\right)\right) \cap W^{1, q}\left(B_{2 R}\left(x_{0}\right)\right)}$, $\|p\|_{W^{1, \infty}\left(B_{2 R}\left(x_{0}\right)\right) \cap W^{2, q}\left(B_{2 R}\left(x_{0}\right)\right)}, \alpha^{*}, C^{*}, q$ and $\|\nabla u\|_{L^{\infty}\left(B_{2 R}\left(x_{0}\right)\right)}$.

Proof. We let $0<R_{0} \leq R, \varepsilon>0$ and define

$$
\begin{aligned}
\lambda_{2 R_{0}}^{*} & =\sup _{B_{2 R_{0}}\left(x_{0}\right)} \lambda^{*}(x), \\
U_{\varepsilon}(x) & =\left(|\nabla u|-\lambda_{2 R_{0}}^{*}-\varepsilon\right)^{+} .
\end{aligned}
$$

Let $0<r \leq R_{0}$. Since for every $\bar{x} \in \overline{B_{2 R_{0}}}\left(x_{0}\right) \cap \partial\{u>0\}$

$$
\limsup _{\substack{x \rightarrow \bar{x} \\ u(x)>0}}|\nabla u| \leq \lambda^{*}(\bar{x}),
$$

then the function $U_{\varepsilon}$ vanishes in a neighborhood of $B_{2 r}\left(x_{0}\right) \cap \partial\{u>0\}$.

We have $|\nabla u| \geq \lambda_{\min }$ in $\left\{U_{\varepsilon}>0\right\}$ and moreover, arguing as in Lemma 3.8 we see that $u \in$ $W^{2, t}\left(B_{2 r}\left(x_{0}\right) \cap\left\{U_{\varepsilon}>0\right\}\right)$ for every $1 \leq t<\infty$. Thus, by Lemma 3.8, $U_{\varepsilon}$ is a solution to

$$
\operatorname{div} D \nabla U_{\varepsilon}+B \cdot \nabla U_{\varepsilon} \geq G
$$

in $\left\{U_{\varepsilon}>0\right\} \cap B_{2 r}\left(x_{0}\right)$ for some functions $D=\left\{D_{i j}\right\}, B=\left\{b_{j}\right\}$ and $G$ such that

$$
\begin{aligned}
& \bar{\beta}|\xi|^{2} \leq \sum_{i j} D_{i j}(x) \xi_{i} \xi_{j} \leq \bar{\beta}^{-1}|\xi|^{2} \quad \text { for every } \xi \in \mathbb{R}^{N}, x \in B_{2 R}\left(x_{0}\right), \\
& \|B\|_{L^{\infty}\left(\left\{U_{\varepsilon}>0\right\} \cap B_{2 R}\left(x_{0}\right)\right)} \leq \bar{C} \quad, \quad\|G\|_{L^{q}\left(\left\{U_{\varepsilon}>0\right\} \cap B_{2 R}\left(x_{0}\right)\right)} \leq \bar{C}
\end{aligned}
$$

with $\bar{\beta}=\bar{\beta}\left(p_{\min }, p_{\max }, \lambda_{\min },\|\nabla u\|_{L^{\infty}\left(B_{2 R}\left(x_{0}\right)\right)}\right), \quad \bar{C}=\bar{C}\left(p_{\min }, p_{\max }, \lambda_{\min },\|\nabla u\|_{L^{\infty}\left(B_{2 R}\left(x_{0}\right)\right)}\right.$, $\left.\|f\|_{L^{\infty}\left(B_{2 R}\left(x_{0}\right)\right) \cap W^{1, q}\left(B_{2 R}\left(x_{0}\right)\right)},\|p\|_{W^{1, \infty}\left(B_{2 R}\left(x_{0}\right)\right) \cap W^{2, q}\left(B_{2 R}\left(x_{0}\right)\right)}\right)$.

Therefore, if $\widetilde{G}$ and $\widetilde{B}$ are the extensions by 0 of $G$ and $B$ respectively from $\left\{U_{\varepsilon}>0\right\} \cap B_{2 r}\left(x_{0}\right)$ to $B_{2 r}\left(x_{0}\right)$ and $\widetilde{D}$ is an extension of $D$ that preserves the uniform ellipticity with the same constants, there holds that $U_{\varepsilon}$ satisfies

$$
\operatorname{div} \widetilde{D} \nabla U_{\varepsilon}+\widetilde{B} \cdot \nabla U_{\varepsilon} \geq \widetilde{G}
$$

in $B_{2 r}\left(x_{0}\right)$ (see, for instance, Lemma 2.1 in [24]).

Let now $h_{\varepsilon}(r)=\sup _{B_{r}\left(x_{0}\right)} U_{\varepsilon}$ and $V=h_{\varepsilon}(2 r)-U_{\varepsilon}$. Then,

$$
\operatorname{div} \widetilde{D} \nabla V+\widetilde{B} \cdot \nabla V \leq-\widetilde{G} \text { in } B_{2 r}\left(x_{0}\right) .
$$

Moreover, $V \geq 0$ in $B_{2 r}\left(x_{0}\right)$. By the weak Harnack inequality (see [17]),

$$
\inf _{B_{r}\left(x_{0}\right)} V+r^{2-N / q}\|\widetilde{G}\|_{L^{q}\left(B_{2 r}\left(x_{0}\right)\right)} \geq c f_{B_{3 r / 2}\left(x_{0}\right)} V
$$

with $c=c\left(N, \bar{\beta},\|\widetilde{B}\|_{L^{\infty}\left(B_{2 R}\left(x_{0}\right)\right)}, q\right)$.

Now, since by the flatness condition, $u$ (and therefore $U_{\varepsilon}$ ) vanishes in the ball $B_{\frac{1-\sigma}{2} r}\left(x_{0}+\frac{1+\sigma}{2} r \nu_{r}\right)$ for some direction $\nu_{r}$, there holds that $V=h_{\varepsilon}(2 r)$ in $B_{\frac{1-\sigma}{2} r}\left(x_{0}+\frac{1+\sigma}{2} r \nu_{r}\right)$ and therefore,

$$
h_{\varepsilon}(2 r)-h_{\varepsilon}(r)+r^{2-N / q} \bar{C} \geq \hat{c}\left(\frac{1-\sigma}{2}\right)^{N} h_{\varepsilon}(2 r) \geq \bar{c} h_{\varepsilon}(2 r)
$$


since $\sigma \leq 1 / 2$, with $\bar{c}=\bar{c}\left(N, \bar{\beta},\|\widetilde{B}\|_{L^{\infty}\left(B_{2 R}\left(x_{0}\right)\right)}, q\right)<1$ and $\bar{C}$ the constant in (3.32). We pass to the limit as $\varepsilon \rightarrow 0$ and we conclude that

$$
h(r) \leq(1-\bar{c}) h(2 r)+r^{2-N / q} \bar{C},
$$

if $r \leq R_{0}$ with $h(r)=\sup _{B_{r}\left(x_{0}\right)}\left(|\nabla u|-\lambda_{2 R_{0}}^{*}\right)^{+}$. Since $2-N / q>0$, there exist $\tilde{\gamma} \in(0,1), \tilde{C}>0$ depending only on $N, q, \bar{c},\|\nabla u\|_{L^{\infty}\left(B_{2 R}\left(x_{0}\right)\right)}$ and $\bar{C}$ such that

$$
h(s) \leq \tilde{C}\left(\frac{s}{2 R_{0}}\right) \tilde{\gamma}
$$

if $s \leq 2 R_{0}$. This implies

$$
\sup _{B_{2 r}\left(x_{0}\right)}|\nabla u| \leq \sup _{B_{2 R_{0}\left(x_{0}\right)}} \lambda^{*}(x)+\tilde{C}\left(\frac{r}{R_{0}}\right)^{\tilde{\gamma}}
$$

if $r \leq R_{0} \leq R$, and the Hölder continuity of $\lambda^{*}(x)$ gives, for $x_{1} \in B_{2 R_{0}}\left(x_{0}\right)$,

$$
\sup _{B_{2 R_{0}\left(x_{0}\right)}} \lambda^{*}(x) \leq \lambda^{*}\left(x_{1}\right)+C^{*}\left(4 R_{0}\right)^{\alpha^{*}} .
$$

We now take $r \leq R, R_{0}=r^{1 / 2} R^{1 / 2}$ and $x_{1} \in B_{r}\left(x_{0}\right)$ and obtain, from (3.35) and (3.36),

$$
\sup _{B_{r}\left(x_{1}\right)}|\nabla u| \leq \sup _{B_{2 r}\left(x_{0}\right)}|\nabla u| \leq \lambda^{*}\left(x_{1}\right)+C\left(\frac{r}{R}\right)^{\gamma},
$$

for $\gamma=\min \left\{\frac{\alpha^{*}}{2}, \frac{\tilde{\gamma}}{2}\right\}$ and $C$ depending only on $\tilde{C}, C^{*}, \tilde{\gamma}$ and $\alpha^{*}$, which proves (3.31) and completes the proof.

Let us show that a point $x_{0}$ in the reduced free boundary of a weak solution is always under the assumptions of Lemma 3.9.

Lemma 3.10. Let $p \in \operatorname{Lip}(\Omega)$ with $1<p_{\min } \leq p(x) \leq p_{\max }<\infty, \lambda^{*} \in C(\Omega)$ with $0<\lambda_{\min } \leq$ $\lambda^{*}(x) \leq \lambda_{\max }<\infty$ and $f \in L^{\infty}(\Omega)$. Let $u$ be a weak solution to $P\left(f, p, \lambda^{*}\right)$ in $\Omega$ and $x_{0} \in$ $\Omega \cap \partial_{\text {red }}\{u>0\}$.

There exists $\sigma_{0}>0$ such that, if $\sigma<\sigma_{0}$, there exists $r_{\sigma}>0$ such that, for every $r \leq r_{\sigma}$,

$$
u \in F(\sigma, 1 ; \infty) \quad \text { in } \quad B_{r}\left(x_{0}\right) \quad \text { in direction } \quad \nu\left(x_{0}\right) \text {, }
$$

with power $p$, slope $\lambda^{*}$ and rhs $f$. Here $\nu\left(x_{0}\right)$ denotes the exterior unit normal to $\Omega \cap \partial\{u>0\}$ at $x_{0}$ in the measure theoretic sense.

Proof. Assume for simplicity that $x_{0}=0$ and $\nu\left(x_{0}\right)=e_{N}$. Let $R>0$ be such that $B_{4 R} \subset \Omega$.

Given $0<\varepsilon<\frac{1}{2}$, there exists $r_{\varepsilon} \leq R$ such that

$$
\frac{\left|\{u>0\} \cap B_{r}^{+}\right|}{\left|B_{r}\right|}<\varepsilon \quad \text { if } \quad r \leq r_{\varepsilon},
$$

and also a constant $c_{N}>1$ so that

$$
\left|B_{r}^{+} \backslash\left\{0<x_{N}<\sigma r\right\}\right| \geq\left|B_{r}\right|\left(1 / 2-c_{N} \sigma\right)>\varepsilon\left|B_{r}\right| \quad \text { if } \quad \sigma<\frac{1 / 2-\varepsilon}{c_{N}} .
$$

Let $r \leq \frac{r_{\varepsilon}}{2}$ and suppose there exists $\bar{x} \in\left(B_{r}^{+} \backslash\left\{0<x_{N}<\sigma r\right\}\right) \cap \partial\{u>0\}$. Then, $\sup _{B_{\rho}(\bar{x})} u \geq c_{\min } \rho$, if $\rho \leq \rho_{0}=\min \left\{r_{0}, R\right\}$, with $c_{\min }$ and $r_{0}$ the constants corresponding to $D=B_{2 R}$ in the definition of weak solution.

Then, if $r \leq \rho_{0}$, there exists $x_{1} \in \bar{B}_{\sigma r / 2}(\bar{x})$ such that $u\left(x_{1}\right) \geq c_{\min } \sigma r / 2$, implying that

$$
u(x) \geq c_{\min } \sigma r / 2-L \kappa \sigma r / 2>0 \quad \text { in } \quad B_{\kappa \sigma r / 2}\left(x_{1}\right) \subset B_{2 r}^{+},
$$


if $\kappa \leq \min \left\{1, \frac{c_{\min }}{2 L}\right\}$, where $L$ is the Lipschitz constant of $u$ in $B_{2 R}$. As a consequence,

$$
\frac{\left|\{u>0\} \cap B_{2 r}^{+}\right|}{\left|B_{2 r}\right|} \geq(\kappa \sigma / 4)^{N}
$$

which contradicts (3.37) if $(\kappa \sigma / 4)^{N}>\varepsilon$. Finally, we fix $\sigma_{0}=\left(2 c_{N}\right)^{-1}$, take $\sigma<\sigma_{0}$ and choose $0<\varepsilon<\frac{1}{2}$ satisfying

$$
\frac{4}{\kappa} \varepsilon^{1 / N}<\sigma<\frac{1 / 2-\varepsilon}{c_{N}} .
$$

Then, letting $r_{\sigma}=\min \left\{\frac{r_{\varepsilon}}{2}, \rho_{0}\right\}$ and $r \leq r_{\sigma}$, we observe that $\left(B_{r}^{+} \backslash\left\{0<x_{N}<\sigma r\right\}\right) \cap \partial\{u>0\}=\emptyset$ by the above discussion, and that we cannot have $u>0$ in $B_{r}^{+} \backslash\left\{0<x_{N}<\sigma r\right\}$ because of (3.37) and (3.38). Therefore we conclude that $u \in F(\sigma, 1 ; \infty)$ in $B_{r}$ with power $p$, slope $\lambda^{*}$ and rhs $f$, for every $r \leq r_{\sigma}$.

Now, we get a result that holds at free boundary points satisfying a density condition on the zero set. This is the situation when $u$ comes from a minimization problem as was the case in $[1,2,8]$, for instance.

Lemma 3.11. Let $p$ and $f$ as in Lemma 3.8 with $q>\max \{1, N / 2\}$ and $\lambda^{*} \in C^{\alpha^{*}}(\Omega)$ with $0<\lambda_{\min } \leq \lambda^{*}(x) \leq \lambda_{\max }<\infty$ in $\Omega$ and $\left[\lambda^{*}\right]_{C^{\alpha^{*}}(\Omega)} \leq C^{*}$. Let $u$ be a weak solution to $P\left(f, p, \lambda^{*}\right)$ in $\Omega$ and let $x_{0} \in \Omega \cap \partial\{u>0\}$ with $B_{4 R}\left(x_{0}\right) \subset \Omega, R \leq 1$. Assume that

$$
\frac{\left|B_{r}\left(x_{0}\right) \cap\{u=0\}\right|}{\left|B_{r}\left(x_{0}\right)\right|} \geq c_{0}>0 \quad \text { if } \quad r \leq R .
$$

Then, for every $x_{1}$ in $B_{r}\left(x_{0}\right)$,

$$
|\nabla u| \leq \lambda^{*}\left(x_{1}\right)+C\left(\frac{r}{R}\right)^{\gamma} \quad \text { in } \quad B_{r}\left(x_{1}\right) \quad \text { if } \quad r \leq R,
$$

for some constants $C$ and $0<\gamma<1$ depending only on $N, p_{\min }, p_{\max }, \lambda_{\min },\|f\|_{L^{\infty}\left(B_{2 R}\left(x_{0}\right)\right) \cap W^{1, q}\left(B_{2 R}\left(x_{0}\right)\right)}$, $\|p\|_{W^{1, \infty}\left(B_{2 R}\left(x_{0}\right)\right) \cap W^{2, q}\left(B_{2 R}\left(x_{0}\right)\right)}, \alpha^{*}, C^{*}, q,\|\nabla u\|_{L^{\infty}\left(B_{2 R}\left(x_{0}\right)\right)}$ and $c_{0}$.

Proof. The proof is exactly as that of Lemma 3.9 the only difference being that instead of the flatness condition we use the density condition (3.39).

Now, with the ideas in the proof of Lemma 3.9 we can improve on the gradient.

Lemma 3.12. Let $p \in W^{1, \infty}\left(B_{\rho}\right) \cap W^{2, q}\left(B_{\rho}\right)$ with $1<p_{\min } \leq p(x) \leq p_{\max }<\infty$ in $B_{\rho}$ and $f \in$ $L^{\infty}\left(B_{\rho}\right) \cap W^{1, q}\left(B_{\rho}\right)$ with $q>\max \{1, N / 2\},\|p\|_{W^{1, \infty}\left(B_{\rho}\right) \cap W^{2, q}\left(B_{\rho}\right)} \leq \widetilde{L}_{1}$ and $\|f\|_{L^{\infty}\left(B_{\rho}\right) \cap W^{1, q}\left(B_{\rho}\right)} \leq$ $\widetilde{L}_{2}$. Let $\lambda^{*} \in C^{\alpha^{*}}\left(B_{\rho}\right)$ with $0<\lambda_{\min } \leq \lambda^{*}(x) \leq \lambda_{\max }<\infty$ in $B_{\rho}$ and $\left[\lambda^{*}\right]_{C^{\alpha^{*}}\left(B_{\rho}\right)} \leq C^{*}$.

Let $0<\theta<1$. There exist $\sigma_{\theta}, c_{\theta}, C, \tilde{C}$ and $\tilde{\gamma}$ such that, if

$$
u \in F(\sigma, 1 ; \tau) \text { in } B_{\rho} \text { in direction } \nu
$$

with power $p$, slope $\lambda^{*}$ and rhs $f$ and, if $\sigma \leq \sigma_{\theta}, \tau \leq \sigma_{\theta} \sigma^{2}$ and $\tilde{C} \rho^{\tilde{\gamma}} \leq \lambda_{\min } \tau$, there holds that

$$
u \in F\left(\theta \sigma, \theta \sigma ; \theta^{2} \tau\right) \text { in } B_{\bar{\rho}} \text { in direction } \bar{\nu}
$$

with the same power, slope and rhs and

$$
c_{\theta} \rho \leq \bar{\rho} \leq \frac{1}{4} \rho, \quad|\nu-\bar{\nu}| \leq C \sigma .
$$

The constants depend only on $N, p_{\min }, p_{\max }, \lambda_{\min }, \lambda_{\max }, \widetilde{L}_{1}, \widetilde{L}_{2}, \alpha^{*}, C^{*}, q$. The constants $\sigma_{\theta}$ and $c_{\theta}$ depend moreover on $\theta$. 
Proof. We will apply Lemma 3.7 inductively, and we will obtain the improvement of the value $\tau$ with an argument similar to the one in Lemma 3.9.

In fact, if $\sigma_{\theta}$ is small enough, we can apply Proposition 3.1 to $\bar{u}(x)=\frac{1}{\rho} u(\rho x)$ and we get

$$
u \in F\left(C_{0} \sigma, C_{0} \sigma ; \tau\right) \text { in } B_{\rho / 2} \text { in direction } \nu,
$$

with power $p$, slope $\lambda^{*}$ and rhs $f$. Then for $0<\theta_{1} \leq \frac{1}{2}$ we can apply Lemma 3.7, if again $\sigma_{\theta}$ is small, and we obtain

$$
u \in F\left(C_{0} \theta_{1} \sigma, 1 ; \tau\right) \text { in } B_{r_{1} \rho} \text { in direction } \nu_{1},
$$

with the same power, slope and rhs, for some $r_{1}, \nu_{1}$ with

$$
c_{\theta_{1}} \leq 2 r_{1} \leq \theta_{1} \text {, and }\left|\nu_{1}-\nu\right| \leq C \sigma .
$$

In order to improve the value of $\tau$ we proceed as in the proof of Lemma 3.9. In fact, we let $R_{0}=R=r_{1} \rho, x_{0}=0$ and repeat the argument leading to (3.34), with $r=r_{1} \rho$. In the present case we use the fact that, because of (3.41), $u$ vanishes in the ball $B_{\frac{r_{1} \rho}{4}}\left(\frac{r_{1} \rho}{2} \nu_{1}\right)$. We also use that, in $B_{\rho},|\nabla u| \leq \lambda^{*}(0)(1+\tau) \leq 2 \lambda_{\max }$. We obtain

$$
\sup _{B_{r_{1} \rho}}\left(|\nabla u|-\lambda_{2 r_{1} \rho}^{*}\right)^{+} \leq(1-\bar{c}) \sup _{B_{2 r_{1} \rho}}\left(|\nabla u|-\lambda_{2 r_{1} \rho}^{*}\right)^{+}+\bar{C}\left(r_{1} \rho\right)^{2-N / q}
$$

with

$$
\lambda_{2 r_{1} \rho}^{*}=\sup _{B_{2 r_{1} \rho}} \lambda^{*}(x),
$$

and constants $0<\bar{c}<1$ and $\bar{C}>0$ depending only on $N, p_{\min }, p_{\max }, \lambda_{\min }, \lambda_{\max }, \widetilde{L}_{1}, \widetilde{L}_{2}$ and $q$. It follows that

$$
\begin{aligned}
\sup _{B_{r_{1} \rho}}|\nabla u| & \leq \lambda_{2 r_{1} \rho}^{*}+(1-\bar{c}) \lambda_{2 r_{1} \rho}^{*} \tau+\bar{C}\left(\frac{\rho}{4}\right)^{2-N / q} \\
& \leq \lambda_{2 r_{1} \rho}^{*}+\left(1-\frac{\bar{c}}{2}\right) \lambda_{2 r_{1} \rho}^{*} \tau
\end{aligned}
$$

if we let $\bar{C}\left(\frac{\rho}{4}\right)^{2-N / q} \leq \frac{\bar{c}}{2} \lambda_{\min } \tau$. Therefore, for $\hat{\theta}=1-\frac{\bar{c}}{2}$, we get

$$
\begin{aligned}
\sup _{B_{r_{1} \rho}}|\nabla u| & \leq \lambda_{2 r_{1} \rho}^{*}(1+\hat{\theta} \tau) \\
& \leq \lambda^{*}(0)(1+\hat{\theta} \tau)+C^{*}\left(2 r_{1} \rho\right)^{\alpha^{*}}(1+\hat{\theta} \tau) \\
& \leq \lambda^{*}(0)\left(1+\hat{\theta} \tau+\frac{1-\hat{\theta}}{2} \tau\right)=\lambda^{*}(0)\left(1+\theta_{0}^{2} \tau\right),
\end{aligned}
$$

if $C^{*} \rho^{\alpha^{*}} \leq \frac{1}{2} \lambda_{\min } \tau$ and $\theta_{1}^{\tilde{\gamma}} \leq \frac{1-\hat{\theta}}{2}$, with $\tilde{\gamma}=\min \left\{\alpha^{*}, 2-N / q\right\}$ and $\theta_{0}=\sqrt{\frac{1+\hat{\theta}}{2}}$.

We see that, if $\theta_{1}$ is chosen small enough,

$$
u \in F\left(\theta_{0} \sigma, 1 ; \theta_{0}^{2} \tau\right) \text { in } B_{r_{1} \rho} \text { in direction } \nu_{1},
$$

with power $p$, slope $\lambda^{*}$ and rhs $f$. Moreover, $r_{1}^{\tilde{\gamma}} \leq \theta_{0}^{2}$.

Then, we can repeat this argument a finite number of times, and we obtain

$$
u \in F\left(\theta_{0}^{m} \sigma, 1 ; \theta_{0}^{2 m} \tau\right) \text { in } B_{r_{1} \ldots r_{m} \rho} \text { in direction } \nu_{m},
$$

with the same power, slope and rhs, with

$$
c_{\theta_{j}} \leq 2 r_{j} \leq \theta_{j} \text {, and }\left|\nu_{m}-\nu\right| \leq \frac{C}{1-\theta_{0}} \sigma .
$$


Finally we choose $m$ large enough and use Proposition 3.1.

\section{Regularity of the Free boundary For WeAK SOlutions to Problem $P\left(f, p, \lambda^{*}\right)$}

In this section we study the regularity of the free boundary for weak solutions to problem $P\left(f, p, \lambda^{*}\right)$.

We prove that the free boundary of a weak solution is a $C^{1, \alpha}$ surface near flat free boundary points (Theorems 4.1, 4.2 and 4.3). As a consequence we get that the free boundary is $C^{1, \alpha}$ in a neighborhood of every point in the reduced free boundary (Theorem 4.4).

We also obtain further regularity results on the free boundary, under further regularity assumptions on the data (Corollary 4.1).

Among Theorems 4.1, 4.2 and 4.3 the most general one is Theorem 4.3.

Theorems 4.1 and 4.2 require the extra assumptions (4.1) and (4.10), respectively. But, under these additional assumptions, the constant in the $C^{1, \alpha}$ continuity of the free boundary becomes universal.

The difference stems from the fact that in Theorems 4.1 and 4.2 the choice of $\rho$ in the statements can be done independently of the weak solution $u$ under consideration, whereas in Theorem 4.3 there is a strong dependence on $u$.

We remark that the Hölder exponent $\alpha$ is universal in the three results.

Our first result holds at free boundary points satisfying a density condition on the zero set. This is the situation when $u$ comes from a minimization problem as was the case in $[1,2,8]$, for instance.

Theorem 4.1. Let $p \in W^{1, \infty}(\Omega) \cap W^{2, q}(\Omega)$ with $1<p_{\min } \leq p(x) \leq p_{\max }<\infty$ in $\Omega$ and $f \in$ $L^{\infty}(\Omega) \cap W^{1, q}(\Omega)$ with $q>\max \{1, N / 2\}$. Let $\lambda^{*} \in C^{\alpha^{*}}(\Omega)$ with $0<\lambda_{\min } \leq \lambda^{*}(x) \leq \lambda_{\max }<\infty$ in $\Omega$ and $\left[\lambda^{*}\right]_{C^{\alpha^{*}}(\Omega)} \leq C^{*}$. Let $u$ be a weak solution to $P\left(f, p, \lambda^{*}\right)$ in $\Omega$ and let $x_{0} \in \Omega \cap \partial\{u>0\}$ with $B_{4 R}\left(x_{0}\right) \subset \Omega, R \leq 1$. Assume that

$$
\frac{\left|B_{r}\left(x_{0}\right) \cap\{u=0\}\right|}{\left|B_{r}\left(x_{0}\right)\right|} \geq c_{0}>0 \quad \text { if } \quad r \leq R .
$$

Then there are constants $\alpha, \beta, \bar{\sigma}_{0}, \bar{C}$ and $C$ such that if

$$
u \in F(\sigma, 1 ; \infty) \text { in } B_{\rho}\left(x_{0}\right) \text { in direction } \nu
$$

with power $p$, slope $\lambda^{*}$ and rhs $f$, with $\sigma \leq \bar{\sigma}_{0}$ and $\bar{C} \rho^{\beta} \leq \bar{\sigma}_{0} \sigma^{2}$, then

$$
B_{\rho / 4}\left(x_{0}\right) \cap \partial\{u>0\} \text { is a } C^{1, \alpha} \text { surface, }
$$

more precisely, a graph in direction $\nu$ of a $C^{1, \alpha}$ function, and, for $x, y$ on this surface,

$$
|\nu(x)-\nu(y)| \leq C \sigma\left|\frac{x-y}{\rho}\right|^{\alpha} .
$$

The constants depend only on $N, p_{\min }, p_{\max }, \lambda_{\min }, \lambda_{\max }, \alpha^{*}, C^{*}, q,\|f\|_{L^{\infty}\left(B_{3 R}\left(x_{0}\right)\right) \cap W^{1, q}\left(B_{3 R}\left(x_{0}\right)\right)}$, $\|p\|_{W^{1, \infty}\left(B_{3 R}\left(x_{0}\right)\right) \cap W^{2, q}\left(B_{3 R}\left(x_{0}\right)\right)}, R, c_{0}$ and the constants $C_{\max }\left(B_{3 R}\left(x_{0}\right)\right)$ and $r_{0}\left(B_{3 R}\left(x_{0}\right)\right)$ in Definition 2.2.

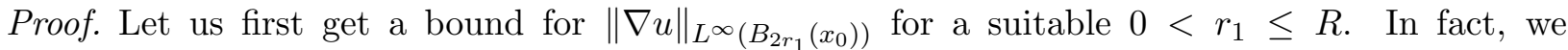
denote $r_{0}=r_{0}\left(B_{3 R}\left(x_{0}\right)\right)$ and $C_{\max }=C_{\max }\left(B_{3 R}\left(x_{0}\right)\right)$, the constants in Definition 2.2. We now let $r_{1}=\frac{1}{4} \min \left\{3 R, r_{0}\right\}$ and see that there holds that $\|u\|_{L^{\infty}\left(B_{4 r_{1}}\left(x_{0}\right)\right)} \leq C_{\max } r_{0}$. 
Then, by Proposition 2.1, it follows that $\|\nabla u\|_{L^{\infty}\left(B_{2 r_{1}}\left(x_{0}\right)\right)}$ can be estimated by a constant depending only on $N, p_{\min }, p_{\max }, r_{1},\|f\|_{L^{\infty}\left(B_{4 r_{1}}\left(x_{0}\right)\right) \cap W^{1, q}\left(B_{4 r_{1}}\left(x_{0}\right)\right)},\|p\|_{W^{1, \infty}\left(B_{4 r_{1}}\left(x_{0}\right)\right) \cap W^{2, q}\left(B_{4 r_{1}}\left(x_{0}\right)\right)}$, $C_{\max }$ and $r_{0}$.

Next, we choose the constants in the statement so that $\rho \leq r_{1}$. Then, we can apply Lemma 3.11 in $B_{4 r_{1}}\left(x_{0}\right)$ and get, for $x \in B_{\rho}\left(x_{0}\right)$,

$$
|\nabla u(x)| \leq \lambda^{*}\left(x_{0}\right)+C_{1} \rho^{\gamma} \leq \lambda^{*}\left(x_{0}\right)\left(1+\frac{C_{1}}{\lambda_{\min }} \rho^{\gamma}\right),
$$

with $C_{1}$ and $\gamma$ constants depending only on $N, p_{\min }, p_{\max }, \lambda_{\min },\|f\|_{L^{\infty}\left(B_{2 r_{1}}\left(x_{0}\right)\right) \cap W^{1, q}\left(B_{2 r_{1}}\left(x_{0}\right)\right)}$, $\|p\|_{W^{1, \infty}\left(B_{2 r_{1}}\left(x_{0}\right)\right) \cap W^{2, q}\left(B_{2 r_{1}}\left(x_{0}\right)\right)}, \alpha^{*}, C^{*}, q,\|\nabla u\|_{L^{\infty}\left(B_{2 r_{1}}\left(x_{0}\right)\right)}, c_{0}$ and $r_{1}$.

We let $\bar{C}$ and $\beta$ in the statement satisfying $\bar{C} \geq \frac{C_{1}}{\lambda_{\min }}$ and $\beta \leq \gamma$, and take $\tau=\bar{C} \rho^{\beta}$. Therefore we obtain

with power $p$, slope $\lambda^{*}$ and rhs $f$.

$$
u \in F(\sigma, 1 ; \tau) \text { in } B_{\rho}\left(x_{0}\right) \text { in direction } \nu,
$$

Applying Proposition 3.1 we have that

$$
u \in F\left(C_{0} \sigma, C_{0} \sigma ; \tau\right) \text { in } B_{\rho / 2}\left(x_{0}\right) \text { in direction } \nu,
$$

with the same power, slope and rhs, if we choose $\bar{C} \geq C^{*}, \beta \leq \alpha^{*}$, and $\bar{\sigma}_{0}$ is small enough so that, in particular, $\tau \leq \sigma$ and $C^{*} \rho^{\alpha^{*}} \leq \bar{C} \rho^{\beta} \leq \lambda_{\min } \sigma$.

Let $x_{1} \in B_{\rho / 2}\left(x_{0}\right) \cap \partial\{u>0\}$. Since Lemma 3.11 also gives

$$
|\nabla u(x)| \leq \lambda^{*}\left(x_{1}\right)+C_{1} \rho^{\gamma} \leq \lambda^{*}\left(x_{1}\right)(1+\tau) \text { in } B_{\rho / 2}\left(x_{1}\right)
$$

and $\left\langle x_{1}-x_{0}, \nu\right\rangle>-C_{0} \sigma \frac{\rho}{2}$ there holds that,

$$
u \in F\left(\bar{C}_{0} \sigma, 1 ; \tau\right) \text { in } B_{\rho / 2}\left(x_{1}\right) \text { in direction } \nu,
$$

with power $p$, slope $\lambda^{*}$ and rhs $f$, for any constant $\bar{C}_{0} \geq\left(C_{0}+2\right)$.

If we let $\bar{\sigma}_{0}$ small enough, the above choice of $\bar{C}$ and $\beta$, which implies in particular that $\tau \leq \bar{C}_{0} \sigma$ and $C^{*}\left(\frac{\rho}{2}\right)^{\alpha^{*}} \leq \lambda_{\min } \bar{C}_{0} \sigma$, allows us to apply again Proposition 3.1 and deduce that

$$
u \in F(C \sigma, C \sigma ; \tau) \text { in } B_{\rho / 4}\left(x_{1}\right) \text { in direction } \nu,
$$

with the same power, slope and rhs.

We want to apply Lemma 3.12 in $B_{\rho / 4}\left(x_{1}\right)$ for some $0<\theta<1$. In fact, we need $C \sigma \leq \sigma_{\theta}$, $\tau \leq \sigma_{\theta}(C \sigma)^{2}$ and $\tilde{C}\left(\frac{\rho}{4}\right)^{\tilde{\gamma}} \leq \lambda_{\min } \tau$, which is satisfied if we let $\bar{\sigma}_{0} \leq \frac{\sigma_{\theta}}{C}, \bar{\sigma}_{0} \leq \sigma_{\theta} C^{2}, \bar{C} \geq \frac{\tilde{C}}{\lambda_{\min }}$ and $\beta \leq \tilde{\gamma}$

Moreover, we want to apply Lemma 3.12 inductively in order to get sequences $\rho_{m}$ and $\nu_{m}$, with $\rho_{0}=\rho / 4$ and $\nu_{0}=\nu$, such that

$$
u \in F\left(\theta^{m} C \sigma, \theta^{m} C \sigma ; \theta^{2 m} \tau\right) \text { in } B_{\rho_{m}}\left(x_{1}\right) \text { in direction } \nu_{m},
$$

with power $p$, slope $\lambda^{*}$ and rhs $f$, with

$$
c_{\theta} \rho_{m} \leq \rho_{m+1} \leq \rho_{m} / 4 \quad \text { and } \quad\left|\nu_{m+1}-\nu_{m}\right| \leq \theta^{m} C \sigma .
$$

For this purpose, we have to verify at each step that

$$
\theta^{m} C \sigma \leq \sigma_{\theta}, \quad \theta^{2 m} \tau \leq \sigma_{\theta}\left(\theta^{m} C \sigma\right)^{2}, \quad \tilde{C} \rho_{m}^{\tilde{\gamma}} \leq \lambda_{\min } \theta^{2 m} \tau .
$$

Since $\rho_{m} \leq 4^{-m} \rho_{0}$, this is satisfied if, in addition, we let $\theta=2^{-\beta}<1$.

Thus, we have that

$$
\left|\left\langle x-x_{1}, \nu_{m}\right\rangle\right| \leq \theta^{m} C \sigma \rho_{m} \quad \text { for } \quad x \in B_{\rho_{m}}\left(x_{1}\right) \cap \partial\{u>0\} .
$$


We also have that there exists $\nu\left(x_{1}\right)=\lim _{m \rightarrow \infty} \nu_{m}$ and

$$
\left|\nu\left(x_{1}\right)-\nu_{m}\right| \leq \frac{C \theta^{m}}{1-\theta} \sigma .
$$

Now let $x \in B_{\rho / 4}\left(x_{1}\right) \cap \partial\{u>0\}$ and choose $m$ such that $\rho_{m+1} \leq\left|x-x_{1}\right|<\rho_{m}$. Then

$$
\left|\left\langle x-x_{1}, \nu\left(x_{1}\right)\right\rangle\right| \leq C \theta^{m} \sigma\left(\frac{\left|x-x_{1}\right|}{1-\theta}+\rho_{m}\right) \leq C \theta^{m} \sigma\left(\frac{1}{1-\theta}+\frac{1}{c_{\theta}}\right)\left|x-x_{1}\right|
$$

and since $\left|x-x_{1}\right| \geq c_{\theta}^{m+1} \rho_{0}$ we have

$$
\theta^{m+1} \leq\left(\frac{\left|x-x_{1}\right|}{\rho_{0}}\right)^{\alpha} \quad \text { with } \quad \alpha=\frac{\beta \log 2}{\log c_{\theta}^{-1}}=\frac{\log \theta}{\log c_{\theta}},
$$

and we obtain that

$$
\left|\left\langle x-x_{1}, \nu\left(x_{1}\right)\right\rangle\right| \leq \frac{C \sigma}{\rho^{\alpha}}\left|x-x_{1}\right|^{1+\alpha}, \quad x \in B_{\rho / 4}\left(x_{1}\right) \cap \partial\{u>0\} .
$$

Let us finally observe that the result in the statement follows if we take $\bar{\sigma}_{0}$ small enough.

In fact, (4.7) implies that $\nu\left(x_{1}\right)$ is the normal to $\partial\{u>0\}$ at $x_{1}$.

From (4.3), (4.7) and (4.5) with $m=0$ we get that $B_{\rho / 4}\left(x_{0}\right) \cap \partial\{u>0\}$ is a graph in the direction $\nu$ of a function $g$ that is defined, differentiable and Lipschitz in $B_{\rho / 4}^{\prime}\left(x_{0}^{\prime}\right)$. This holds if $\bar{\sigma}_{0}$ is small so that

$$
\sqrt{1-\left(C_{0} \sigma\right)^{2}} \geq 1 / 2 \quad \text { and } \quad C \sigma\left(1+\frac{1}{1-\theta}\right) \leq 1 / 2 \quad \text { for } \quad \sigma \leq \bar{\sigma}_{0}
$$

With these choices, the Lipschitz constant of $g$ is universal (observe that (4.3) implies that $\left|g\left(x^{\prime}\right)-g\left(x_{1}^{\prime}\right)\right| \leq C_{0} \sigma \rho$ if $\left.x^{\prime}, x_{1}^{\prime} \in B_{\rho / 4}^{\prime}\left(x_{0}^{\prime}\right)\right)$.

In order to see that (4.2) holds we let $x, y \in B_{\rho / 2}\left(x_{0}\right) \cap \partial\{u>0\}$ such that $|x-y|<\rho / 8$.

We can apply the construction above with $x_{1}=y$, so we have sequences $\rho_{m}=\rho_{m}(y)$ with $\rho_{0}(y)=\rho / 4$, and $\nu_{m}=\nu_{m}(y)$ satisfying (4.4), with $\nu(y)=\lim _{m \rightarrow \infty} \nu_{m}(y)$.

Now let $m_{0}$ be such that

$$
\frac{\rho_{m_{0}+1}}{2} \leq|x-y|<\frac{\rho_{m_{0}}}{2}
$$

We use that

$$
u \in F\left(\sigma_{m_{0}}, \sigma_{m_{0}} ; \tau_{m_{0}}\right) \text { in } B_{\rho_{m_{0}}}(y) \text { in direction } \nu_{m_{0}}(y),
$$

with power $p$, slope $\lambda^{*}$ and rhs $f$, for $\sigma_{m_{0}}=\theta^{m_{0}} C \sigma$ and $\tau_{m_{0}}=\theta^{2 m_{0}} \tau$.

In fact, we have now the following picture: $u$ is under the assumption of the theorem with $x_{0}$ replaced by $y$ and flatness condition (4.9). Then, with $x_{1}$ replaced by $x, \rho_{0}(x)=\rho_{m_{0}}(y)$ and $\nu_{0}(x)=\nu_{m_{0}}(y),(4.5)$ with $m=0$ gives

$$
\left|\nu(x)-\nu_{m_{0}}(y)\right|=\left|\nu(x)-\nu_{0}(x)\right| \leq \frac{C \sigma_{m_{0}}}{1-\theta} .
$$

Let us notice that, from the choice of $\alpha$ we made in (4.6), $\sigma_{m_{0}}=C \sigma \theta^{m_{0}}=C \sigma\left(c_{\theta}^{m_{0}}\right)^{\alpha}$. Since, by (4.4) and (4.8), $c_{\theta}^{m_{0}+1} \leq 4 \frac{\rho_{m_{0}+1}}{\rho} \leq \frac{8}{\rho}|x-y|$, there holds

$$
\left|\nu(x)-\nu_{m_{0}}(y)\right| \leq \frac{C \sigma}{1-\theta}\left(\frac{8|x-y|}{c_{\theta} \rho}\right)^{\alpha} .
$$


Estimate (4.5) also gives

We thus get

$$
\left|\nu(y)-\nu_{m_{0}}(y)\right| \leq \frac{C \sigma}{1-\theta}\left(\frac{8|x-y|}{c_{\theta} \rho}\right)^{\alpha}
$$

$$
|\nu(x)-\nu(y)| \leq C \sigma\left|\frac{x-y}{\rho}\right|^{\alpha} \quad \text { if } \quad x, y \in B_{\rho / 2}\left(x_{0}\right) \cap \partial\{u>0\}, \quad|x-y|<\rho / 8 .
$$

Finally, if $x, y \in B_{\rho / 4}\left(x_{0}\right) \cap \partial\{u>0\}$ are such that $|x-y| \geq \rho / 8$ we can find points $z_{i} \in$ $B_{\rho / 4}\left(x_{0}\right) \cap \partial\{u>0\}$ with $z_{0}=x, z_{k}=y,\left|z_{i}-z_{i+1}\right|<\rho / 8$ for every $i$ and $k$ a universal number. By applying the last estimate we get (4.2).

So, the theorem is proved.

In the next result we replace the density condition (4.1) of Theorem 4.1 by a flatness condition at the point, at every scale. In fact, we get

Theorem 4.2. Let $p \in W^{1, \infty}(\Omega) \cap W^{2, q}(\Omega)$ with $1<p_{\min } \leq p(x) \leq p_{\max }<\infty$ in $\Omega$ and $f \in$ $L^{\infty}(\Omega) \cap W^{1, q}(\Omega)$ with $q>\max \{1, N / 2\}$. Let $\lambda^{*} \in C^{\alpha^{*}}(\Omega)$ with $0<\lambda_{\min } \leq \lambda^{*}(x) \leq \lambda_{\max }<\infty$ in $\Omega$ and $\left[\lambda^{*}\right]_{C^{\alpha^{*}}(\Omega)} \leq C^{*}$. Let $u$ be a weak solution to $P\left(f, p, \lambda^{*}\right)$ in $\Omega$ and let $x_{0} \in \Omega \cap \partial\{u>0\}$ with $B_{4 R}\left(x_{0}\right) \subset \Omega, R \leq 1$. Assume that, for every $r \leq R$,

$$
u \in F(1 / 2,1 ; \infty) \text { in } B_{r}\left(x_{0}\right) \text { in some direction } \nu_{r},
$$

with power $p$, slope $\lambda^{*}$ and rhs $f$.

Then there are constants $\alpha, \beta, \bar{\sigma}_{0}, \bar{C}$ and $C$ such that if

$$
u \in F(\sigma, 1 ; \infty) \text { in } B_{\rho}\left(x_{0}\right) \text { in direction } \nu
$$

with power $p$, slope $\lambda^{*}$ and rhs $f$, with $\sigma \leq \bar{\sigma}_{0}$ and $\bar{C} \rho^{\beta} \leq \bar{\sigma}_{0} \sigma^{2}$, then

$$
B_{\rho / 4}\left(x_{0}\right) \cap \partial\{u>0\} \text { is a } C^{1, \alpha} \text { surface, }
$$

more precisely, a graph in direction $\nu$ of a $C^{1, \alpha}$ function, and, for $x, y$ on this surface,

$$
|\nu(x)-\nu(y)| \leq C \sigma\left|\frac{x-y}{\rho}\right|^{\alpha} .
$$

The constants depend only on $N, p_{\min }, p_{\max }, \lambda_{\min }, \lambda_{\max }, \alpha^{*}, C^{*}, q,\|f\|_{L^{\infty}\left(B_{3 R}\left(x_{0}\right)\right) \cap W^{1, q}\left(B_{3 R}\left(x_{0}\right)\right)}$, $\|p\|_{W^{1, \infty}\left(B_{3 R}\left(x_{0}\right)\right) \cap W^{2, q}\left(B_{3 R}\left(x_{0}\right)\right)}, R$ and the constants $C_{\max }\left(B_{3 R}\left(x_{0}\right)\right)$ and $r_{0}\left(B_{3 R}\left(x_{0}\right)\right)$ in Definition 2.2.

Proof. The proof is exactly as that of Theorem 4.1 the only difference being that instead of using Lemma 3.11, we make use of Lemma 3.9.

Our last result on the regularity of the free boundary of a weak solution in a neighborhood of a flat free boundary point holds without the extra assumptions (4.1) and (4.10) of Theorems 4.1 and 4.2. In fact, we get

Theorem 4.3. Let $p \in W^{1, \infty}(\Omega) \cap W^{2, q}(\Omega)$ with $1<p_{\min } \leq p(x) \leq p_{\max }<\infty$ in $\Omega$ and $f \in$ $L^{\infty}(\Omega) \cap W^{1, q}(\Omega)$ with $q>\max \{1, N / 2\}$. Let $\lambda^{*} \in C^{\alpha^{*}}(\Omega)$ with $0<\lambda_{\min } \leq \lambda^{*}(x) \leq \lambda_{\max }<\infty$ in $\Omega$ and $\left[\lambda^{*}\right]_{C^{\alpha^{*}}(\Omega)} \leq C^{*}$. Let $u$ be a weak solution to $P\left(f, p, \lambda^{*}\right)$ in $\Omega$ and let $x_{0} \in \Omega \cap \partial\{u>0\}$.

Then there are constants $\alpha, \bar{\sigma}_{0}$ and $C$ such that if

$$
u \in F(\sigma, 1 ; \infty) \text { in } B_{\rho}\left(x_{0}\right) \text { in direction } \nu
$$


with power $p$, slope $\lambda^{*}$ and rhs $f$, with $\sigma \leq \bar{\sigma}_{0}$ and $\rho$ small enough, then

$$
B_{\rho / 4}\left(x_{0}\right) \cap \partial\{u>0\} \text { is a } C^{1, \alpha} \text { surface, }
$$

more precisely, a graph in direction $\nu$ of a $C^{1, \alpha}$ function, and, for $x, y$ on this surface,

$$
|\nu(x)-\nu(y)| \leq C \sigma\left|\frac{x-y}{\rho}\right|^{\alpha} .
$$

The constants $\alpha, \bar{\sigma}_{0}$ and $C$ depend only on $N, p_{\min }, p_{\max },\|f\|_{L^{\infty}(\Omega) \cap W^{1, q}(\Omega)},\|p\|_{W^{1, \infty}(\Omega) \cap W^{2, q}(\Omega)}$, $\lambda_{\min }, \lambda_{\max }, \alpha^{*}, C^{*}$ and $q$.

Proof. Since

$$
\limsup _{\substack{x \rightarrow x_{0} \\ u(x)>0}}|\nabla u(x)| \leq \lambda^{*}\left(x_{0}\right),
$$

given $\bar{\sigma}_{0}$ and $\sigma \leq \bar{\sigma}_{0}$, there exists $\rho_{1}=\rho_{1}\left(u, x_{0}, \bar{\sigma}_{0}, \sigma, \lambda_{\min }\right)$ such that, if $\rho \leq \rho_{1}$,

$$
|\nabla u(x)| \leq \lambda^{*}\left(x_{0}\right)\left(1+\frac{\bar{\sigma}_{0} \sigma^{2}}{2}\right), \quad \text { for } x \in B_{\rho}\left(x_{0}\right) .
$$

We take $\tau=\bar{\sigma}_{0} \sigma^{2}$ and obtain

$$
u \in F(\sigma, 1 ; \tau) \text { in } B_{\rho}\left(x_{0}\right) \text { in direction } \nu,
$$

with power $p$, slope $\lambda^{*}$ and rhs $f$.

Applying Proposition 3.1 we have that

$$
u \in F\left(C_{0} \sigma, C_{0} \sigma ; \tau\right) \text { in } B_{\rho / 2}\left(x_{0}\right) \text { in direction } \nu,
$$

with the same power, slope and rhs, if $\bar{\sigma}_{0}$ is small enough so that, in particular, $\tau \leq \sigma$ and $\rho \leq \rho_{2}\left(C^{*}, \alpha^{*}, \lambda_{\min }, \sigma\right)$ so that $C^{*} \rho^{\alpha^{*}} \leq \lambda_{\min } \sigma$.

Let $x_{1} \in B_{\rho / 2}\left(x_{0}\right) \cap \partial\{u>0\}$. From (4.11) and the Hölder continuity of $\lambda^{*}(x)$ we get

$$
|\nabla u(x)| \leq\left(\lambda^{*}\left(x_{1}\right)+C^{*}(\rho / 2)^{\alpha^{*}}\right)\left(1+\frac{\bar{\sigma}_{0} \sigma^{2}}{2}\right) \leq \lambda^{*}\left(x_{1}\right)(1+\tau) \quad \text { in } B_{\rho / 2}\left(x_{1}\right),
$$

if $\rho \leq \rho_{3}\left(C^{*}, \alpha^{*}, \lambda_{\min }, \bar{\sigma}_{0}, \sigma\right)$, so that $C^{*}(\rho / 2)^{\alpha^{*}} \leq \lambda_{\min } \frac{\bar{\sigma}_{0} \sigma^{2}}{4}$.

Then,

$$
u \in F\left(\bar{C}_{0} \sigma, 1 ; \tau\right) \text { in } B_{\rho / 2}\left(x_{1}\right) \text { in direction } \nu,
$$

with power $p$, slope $\lambda^{*}$ and rhs $f$, for any constant $\bar{C}_{0} \geq C_{0}+2$.

If we let $\bar{\sigma}_{0}$ small enough, so that, in particular, $\tau \leq \bar{C}_{0} \sigma$, and take $\rho \leq \rho_{4}\left(C^{*}, \alpha^{*}, \lambda_{\min }, \bar{C}_{0}, \sigma\right)$ so that $C^{*}\left(\frac{\rho}{2}\right)^{\alpha^{*}} \leq \lambda_{\min } \bar{C}_{0} \sigma$, we can apply again Proposition 3.1 and deduce that

$$
u \in F(C \sigma, C \sigma ; \tau) \text { in } B_{\rho / 4}\left(x_{1}\right) \text { in direction } \nu,
$$

with the same power, slope and rhs.

We want to apply Lemma 3.12 in $B_{\rho / 4}\left(x_{1}\right)$ for some $0<\theta<1$. In fact, we need $C \sigma \leq \sigma_{\theta}$, $\tau \leq \sigma_{\theta}(C \sigma)^{2}$ and $\tilde{C}\left(\frac{\rho}{4}\right)^{\tilde{\gamma}} \leq \lambda_{\min } \tau$, which is satisfied if we let $\bar{\sigma}_{0} \leq \frac{\sigma_{\theta}}{C}, \bar{\sigma}_{0} \leq \sigma_{\theta} C^{2}$ and $\rho \leq$ $\rho_{5}\left(\tilde{C}, \tilde{\gamma}, \lambda_{\min }, \bar{\sigma}_{0}, \sigma\right)$.

Moreover, we want to apply Lemma 3.12 inductively in order to get sequences $\rho_{m}$ and $\nu_{m}$, with $\rho_{0}=\rho / 4$ and $\nu_{0}=\nu$, such that

$$
u \in F\left(\theta^{m} C \sigma, \theta^{m} C \sigma ; \theta^{2 m} \tau\right) \text { in } B_{\rho_{m}}\left(x_{1}\right) \text { in direction } \nu_{m},
$$

with power $p$, slope $\lambda^{*}$ and rhs $f$, with $c_{\theta} \rho_{m} \leq \rho_{m+1} \leq \rho_{m} / 4$ and $\left|\nu_{m+1}-\nu_{m}\right| \leq \theta^{m} C \sigma$. 
For this purpose, we have to verify at each step

$$
\theta^{m} C \sigma \leq \sigma_{\theta}, \quad \theta^{2 m} \tau \leq \sigma_{\theta}\left(\theta^{m} C \sigma\right)^{2}, \quad \tilde{C} \rho_{m}^{\tilde{\gamma}} \leq \lambda_{\min } \theta^{2 m} \tau .
$$

Since $\rho_{m} \leq 4^{-m} \rho_{0}$, this is satisfied if, in addition, we let $\theta=2^{-\tilde{\gamma}}<1$.

Now the proof follows as that of Theorem 4.1, with $\alpha=\frac{\tilde{\gamma} \log 2}{\log c_{\theta}^{-1}}$, and the conclusion is obtained if $\rho \leq \bar{\rho}_{0}=\min \left\{\rho_{1}, \rho_{2}, \rho_{3}, \rho_{4}, \rho_{5}\right\}$.

As a consequence of Theorem 4.3 we obtain

Theorem 4.4. Let $f, p$ and $\lambda^{*}$ be as in Theorem 4.3. Let $u$ be a weak solution of $P\left(f, p, \lambda^{*}\right)$ in $\Omega$ and let $x_{0} \in \Omega \cap \partial_{\text {red }}\{u>0\}$. There exists $\bar{r}_{0}>0$ such that $B_{\bar{r}_{0}}\left(x_{0}\right) \cap \partial\{u>0\}$ is a $C^{1, \alpha}$ surface for some $0<\alpha<1$. It follows that, for some $0<\gamma<1$, $u$ is $C^{1, \gamma}$ up to $B_{\bar{r}_{0}}\left(x_{0}\right) \cap \partial\{u>0\}$ and the free boundary condition is satisfied in the classical sense. In addition, for every $x_{1} \in B_{\bar{r}_{0}}\left(x_{0}\right) \cap \partial\{u>0\}$ there is a neighborhood $\mathcal{U}$ such that $\nabla u \neq 0$ in $\mathcal{U} \cap\{u>0\}, u \in W_{\text {loc }}^{2,2}(\mathcal{U} \cap\{u>0\})$ and the equation is satisfied in a pointwise sense in $\mathcal{U} \cap\{u>0\}$.

If moreover $\nabla p$ and $f$ are Hölder continuous in $\Omega$, then $u \in C^{2}(\mathcal{U} \cap\{u>0\})$ and the equation is satisfied in the classical sense in $\mathcal{U} \cap\{u>0\}$.

Proof. The result follows from Theorem 4.3, by applying Lemma 3.10 at the point $x_{0}$.

The $C^{1, \gamma}$ smoothness of $u$ up to $\partial\{u>0\}$, for some $0<\gamma<1$, follows from the regularity results up to the boundary of [14] (see Theorem 1.2 in [14]).

We can also obtain higher regularity of $\partial\{u>0\}$ if the data are smoother. We have

Corollary 4.1. Let $u, x_{0}$ and $\bar{r}_{0}$ be as in Theorem 4.4. Assume moreover that $p \in C^{2}(\Omega)$, $f \in C^{1}(\Omega)$ and $\lambda^{*} \in C^{2}(\Omega)$, then $B_{\bar{r}_{0}}\left(x_{0}\right) \cap \partial\{u>0\} \in C^{2, \mu}$ for every $0<\mu<1$. If $p \in C^{m+1, \mu}(\Omega)$, $f \in C^{m, \mu}(\Omega)$ and $\lambda^{*} \in C^{m+1, \mu}(\Omega)$ for some $0<\mu<1$ and $m \geq 1$, then $B_{\bar{r}_{0}}\left(x_{0}\right) \cap \partial\{u>0\} \in$ $C^{m+2, \mu}$.

Finally, if $p, f$ and $\lambda^{*}$ are analytic, then $B_{\bar{r}_{0}}\left(x_{0}\right) \cap \partial\{u>0\}$ is analytic.

Proof. As in Theorem 8.4 in [1], Theorem 6.3 and Remark 6.4 in [2] and Corollary 9.2 in [8], we use Theorem 2 in [19].

In fact, we apply this theorem with our equation seen in the form $F\left(x, u, D u, D^{2} u\right)=0$, with

$$
F(x, s, q, M)=|q|^{p(x)-2}\left[\sum_{i j}\left(\delta_{i j}+(p(x)-2) \frac{q_{i} q_{j}}{|q|^{2}}\right) M_{i j}+\sum_{j} p_{x_{j}}(x) \log |q| q_{j}\right]-f(x),
$$

in a neighborhood of the free boundary where $|\nabla u| \geq \frac{\lambda_{\min }}{2}$, and boundary condition in the form $g(x, D u)=0$, with

$$
g(x, q)=|q|^{2}-\lambda^{* 2}(x) .
$$

Already in [1] it was observed that Theorem 2 in [19] holds with $u \in C^{2}$ in $\{u>0\}$ and $u \in C^{1, \gamma}$ up to $\partial\{u>0\}$, even though the result in [19] is stated with $u \in C^{2}$ up to $\partial\{u>0\}$.

\section{Application to a singular perturbation problem}

In this section we apply the regularity results obtained in the previous section to a singular perturbation problem we studied in [25]. Our regularity results apply to limit functions satisfying suitable conditions that are fulfilled, for instance, under the situation we considered in [26].

For a different application of these regularity results we refer to our work [26].

We next consider the following singular pertubation problem for the $p_{\varepsilon}(x)$-Laplacian:

$$
\left(P_{\varepsilon}\left(f^{\varepsilon}, p_{\varepsilon}\right)\right) \quad \Delta_{p_{\varepsilon}(x)} u^{\varepsilon}=\beta_{\varepsilon}\left(u^{\varepsilon}\right)+f^{\varepsilon}, \quad u^{\varepsilon} \geq 0
$$


in a domain $\Omega \subset \mathbb{R}^{N}$. Here $\varepsilon>0, \beta_{\varepsilon}(s)=\frac{1}{\varepsilon} \beta\left(\frac{s}{\varepsilon}\right)$, with $\beta$ a Lipschitz function satisfying $\beta>0$ in $(0,1), \beta \equiv 0$ outside $(0,1)$ and $\int \beta(s) d s=M$.

We assume that $1<p_{\min } \leq p_{\varepsilon}(x) \leq p_{\max }<\infty,\left\|\nabla p_{\varepsilon}\right\|_{L^{\infty}} \leq L$ and that the functions $u^{\varepsilon}$ and $f^{\varepsilon}$ are uniformly bounded.

In [25] we proved local uniform Lipschitz regularity for solutions of this problem, we passed to the limit $(\varepsilon \rightarrow 0)$ and we showed that, under suitable assumptions, limit functions are weak solutions to the free boundary problem: $u \geq 0$ and

$\left(P\left(f, p, \lambda^{*}\right)\right) \quad \begin{cases}\Delta_{p(x)} u=f & \text { in }\{u>0\} \\ u=0,|\nabla u|=\lambda^{*}(x) & \text { on } \partial\{u>0\}\end{cases}$

with $\lambda^{*}(x)=\left(\frac{p(x)}{p(x)-1} M\right)^{1 / p(x)}, p=\lim p_{\varepsilon}$ and $f=\lim f^{\varepsilon}$.

Before giving the precise statement of one of the results we proved in [25], we need the following definitions

Definition 5.1. Let $u$ be a continuous nonnegative function in a domain $\Omega \subset \mathbb{R}^{N}$. Let $x_{0} \in$ $\Omega \cap \partial\{u>0\}$. We say that $x_{0}$ is a regular point from the positive side if there is a ball $B \subset\{u>0\}$ with $x_{0} \in \partial B$.

Definition 5.2. Let $u$ be a continuous nonnegative function in a domain $\Omega \subset \mathbb{R}^{N}$. Let $x_{0} \in$ $\Omega \cap \partial\{u>0\}$.

We say that condition (D) holds at $x_{0}$ if there exist $\gamma>0$ and $0<c<1$ such that, for every $x \in B_{\gamma}\left(x_{0}\right) \cap \partial\{u>0\}$ which is regular from the positive side and $r \leq \gamma$, there holds that $\left|\{u=0\} \cap B_{r}(x)\right| \geq c\left|B_{r}(x)\right|$.

Definition 5.3. Let $u$ be a continuous nonnegative function in a domain $\Omega \subset \mathbb{R}^{N}$. Let $x_{0} \in$ $\Omega \cap \partial\{u>0\}$.

We say that condition (L) holds at $x_{0}$ if there exist $\gamma>0, \theta>0$ and $s_{0}>0$ such that for every point $y \in B_{\gamma}\left(x_{0}\right) \cap \partial\{u>0\}$ which is regular from the positive side, and for every ball $B_{r}(z) \subset\{u>0\}$ with $y \in \partial B_{r}(z)$ and $r \leq \gamma$, there exists a unit vector $\tilde{e}_{y}$, with $\left\langle\tilde{e}_{y}, z-y\right\rangle>\theta\|z-y\|$, such that $u\left(y-s \tilde{e}_{y}\right)=0$ for $0<s<s_{0}$.

In [25] we obtained the following result:

Theorem 5.1. Let $u^{\varepsilon_{j}}$ be a family of solutions to $P_{\varepsilon_{j}}\left(f^{\varepsilon_{j}}, p_{\varepsilon_{j}}\right)$ in a domain $\Omega \subset \mathbb{R}^{N}$ with $1<$ $p_{\min } \leq p_{\varepsilon_{j}}(x) \leq p_{\max }<\infty$ and $p_{\varepsilon_{j}}(x)$ Lipschitz continuous with $\left\|\nabla p_{\varepsilon_{j}}\right\|_{L^{\infty}} \leq L$, for some $L>0$. Assume that $u^{\varepsilon_{j}} \rightarrow u$ uniformly on compact subsets of $\Omega, f^{\varepsilon_{j}} \rightarrow f *-$ weakly in $L^{\infty}(\Omega), p_{\varepsilon_{j}} \rightarrow p$ uniformly on compact subsets of $\Omega$ and $\varepsilon_{j} \rightarrow 0$.

Assume that $u$ is locally uniformly nondegenerate on $\Omega \cap \partial\{u>0\}$ and that at every point $x_{0} \in \Omega \cap \partial\{u>0\}$ either condition $(D)$ or condition $(L)$ holds.

Then, $u$ is a weak solution to the free boundary problem: $u \geq 0$ and

$$
\begin{aligned}
& \left(P\left(f, p, \lambda^{*}\right)\right) \quad \begin{cases}\Delta_{p(x)} u=f & \text { in }\{u>0\} \\
u=0,|\nabla u|=\lambda^{*}(x) & \text { on } \partial\{u>0\}\end{cases} \\
& \text { with } \lambda^{*}(x)=\left(\frac{p(x)}{p(x)-1} M\right)^{1 / p(x)} \text { and } M=\int \beta(s) d s .
\end{aligned}
$$


Remark 5.1. In [26] we proved that if $u^{\varepsilon_{j}}, f^{\varepsilon_{j}}, p_{\varepsilon_{j}}, \varepsilon_{j}, f$ and $p$ are as in Theorem 5.1 and $u^{\varepsilon_{j}} \rightarrow u$ uniformly on compact subsets of $\Omega$ with $u^{\varepsilon_{j}}$ local minimizers of an energy functional, then $u$ is under the assumptions of Theorem 5.1.

As a first application of Theorem 4.4 we obtain the following result on the regularity of the free boundary for limit functions of the singular perturbation problem $P_{\varepsilon_{j}}\left(f^{\varepsilon_{j}}, p_{\varepsilon_{j}}\right)$.

Theorem 5.2. Let $u^{\varepsilon_{j}}, f^{\varepsilon_{j}}, p_{\varepsilon_{j}}, \varepsilon_{j}, u, f$ and $p$ be as in Theorem 5.1. Assume moreover that $f \in W^{1, q}(\Omega)$ and $p \in W^{2, q}(\Omega)$ with $q>\max \{1, N / 2\}$.

Let $x_{0} \in \Omega \cap \partial_{\text {red }}\{u>0\}$. Then, there exists $\bar{r}_{0}>0$ such that $B_{\bar{r}_{0}}\left(x_{0}\right) \cap \partial\{u>0\}$ is a $C^{1, \alpha}$ surface for some $0<\alpha<1$. It follows that, for some $0<\gamma<1$, $u$ is $C^{1, \gamma}$ up to $B_{\bar{r}_{0}}\left(x_{0}\right) \cap \partial\{u>0\}$ and the free boundary condition is satisfied in the classical sense. In addition, for every $x_{1} \in B_{\bar{r}_{0}}\left(x_{0}\right) \cap \partial\{u>$ $0\}$ there is a neighborhood $\mathcal{U}$ such that $\nabla u \neq 0$ in $\mathcal{U} \cap\{u>0\}, u \in W_{\text {loc }}^{2,2}(\mathcal{U} \cap\{u>0\})$ and the equation is satisfied in a pointwise sense in $\mathcal{U} \cap\{u>0\}$.

If moreover $\nabla p$ and $f$ are Hölder continuous in $\Omega$, then $u \in C^{2}(\mathcal{U} \cap\{u>0\})$ and the equation is satisfied in the classical sense in $\mathcal{U} \cap\{u>0\}$.

Proof. The result follows from the application of Theorems 5.1 and 4.4 above.

We also obtain higher regularity from the application of Corollary 4.1.

Corollary 5.1. Let $u, x_{0}$ and $\bar{r}_{0}$ be as in Theorem 5.2. Assume moreover that $p \in C^{2}(\Omega)$ and $f \in C^{1}(\Omega)$, then $B_{\bar{r}_{0}}\left(x_{0}\right) \cap \partial\{u>0\} \in C^{2, \mu}$ for every $0<\mu<1$. If $p \in C^{m+1, \mu}(\Omega)$ and $f \in C^{m, \mu}(\Omega)$ for some $0<\mu<1$ and $m \geq 1$, then $B_{\bar{r}_{0}}\left(x_{0}\right) \cap \partial\{u>0\} \in C^{m+2, \mu}$.

Finally, if $p$ and $f$ are analytic, then $B_{\bar{r}_{0}}\left(x_{0}\right) \cap \partial\{u>0\}$ is analytic.

\section{REFERENCES}

[1] Alt, H. W., \& Caffarelli, L. A. Existence and regularity for a minimum problem with free boundary, J. Reine Angew. Math. 325 (1981), 105-144.

[2] Alt, H. W., Caffarelli, L. A., \& Friedman, A. A free boundary problem for quasilinear elliptic equations, Ann. Sc. Norm. Super. Pisa Cl. Sci. (4) 11 (1) (1984), 1-44.

[3] Caffarelli, L. A. A Harnack inequality approach to the regularity of free boundaries. Part I: Lipschitz free boundaries are $C^{1, \alpha}$, Rev. Mat. Iberoam. 3 (2) (1987), 139-162.

[4] Caffarelli, L. A. A Harnack inequality approach to the regularity of free boundaries. Part II: Flat free boundaries are Lipschitz, Comm. Pure Appl. Math. 42 (1989), 55-78.

[5] Caffarelli, L. A., Jerison, D., \& Kenig, C. E. Global energy minimizers for free boundary problems and full regularity in three dimensions, Contemp. Math. 350 (2004), 83-97.

[6] Challal, S., \& Lyaghfouri, A. Gradient estimates for p (x)-harmonic functions, Manuscripta Math. 131 (2010), 403-414.

[7] Challal, S., \& Lyaghfouri, A. Second order regularity for the $p(x)$-Laplace operator, Math. Nachr. 284 (10) (2011), 1270-1279.

[8] Danielli, D., \& Petrosyan, A. A minimum problem with free boundary for a degenerate quasilinear operator, Calc. Var. Partial Differential Equations 23 (1) (2005), 97-124.

[9] Danielli, D., \& Petrosyan, A. Full regularity of the free boundary in a Bernoulli-type problem in two dimensions, Math. Res. Lett. 13 (2006), 667-681.

[10] De Silva, D. Free boundary regularity for a problem with right hand side, Interfaces Free Bound. 13 (2011), 223-238.

[11] De Silva, D., Ferrari, F., \& Salsa, S. Free boundary regularity for fully nonlinear nonhomogeneous two phase problems, J. Math. Pures Appl. 103 (3) (2015), 658-694.

[12] Diening, L., Harjulehto, P., Hasto, P., \& Ruzicka, M. Lebesgue and Sobolev Spaces with Variable Exponents, Lecture Notes in Math. 2017, Springer, 2011. 
[13] Evans, L. C., \& Gariepy, F. Measure Theory and Fine Properties of Functions, CRC Press, Boca RatonLondon-New York- Washigton DC, 1992.

[14] Fan, X. Global $C^{1, \alpha}$ regularity for variable exponent elliptic equations in divergence form, J. Differential Equations 235 (2007), 397-417.

[15] Federer, H. Geometric Measure Theory, Die Grundlehren der mathematischen Wissenschaften, Band 153, Springer-Verlag New York Inc., New York, 1969.

[16] Fernandez Bonder, J., Martínez, S., \& Wolanski, N. A free boundary problem for the p $(x)$-Laplacian, Nonlinear Anal. 72 (2010), 1078-1103.

[17] Gilbarg, D., \& Trudinger, N. S. Elliptic Partial Differential Equations of Second Order, Grundlehren der Mathematischen Wissenschaften [Fundamental Principles of Mathematical Sciences], vol. 224, Springer-Verlag, Berlin, 1983.

[18] Gustafsson, B., \& Shahgholian, H. Existence and geometric properties of solutions of a free boundary problem in potential theory, J. Reine Angew. Math. 473 (1996), 137-179.

[19] Kinderlehrer, D., \& Nirenberg, L. Regularity in free boundary problems, Ann. Sc. Norm. Super. Pisa Cl. Sci. (4) 4 (2) (1977), 373-391.

[20] Kováčik, O., \& Rákosník, J. On spaces $L^{p(x)}$ and $W^{k, p(x)}$, Czechoslovak Math. J. 41 (1991), 592-618.

[21] Lederman, C. A free boundary problem with a volume penalization, Ann. Sc. Norm. Super. Pisa Cl. Sci. (4) 23 (2) (1996), 249-300.

[22] Lederman, C., \& Wolanski, N. Viscosity solutions and regularity of the free boundary for the limit of an elliptic two phase singular perturbation problem, Ann. Sc. Norm. Super. Pisa Cl. Sci. (4) 27 (2) (1998), 253-288.

[23] Lederman, C., \& Wolanski, N. Singular perturbation in a nonlocal diffusion problem, Comm. Partial Differential Equations 31 (2) (2006), 195-241.

[24] Lederman, C., \& Wolanski, N. A two phase elliptic singular perturbation problem with a forcing term, J. Math. Pures Appl. 86 (6) (2006), 552-589.

[25] Lederman, C., \& Wolanski, N. An inhomogeneous singular perturbation problem for the $p(x)$-Laplacian, Nonlinear Anal. 138 (2016), 300-325.

[26] Lederman, C., \& Wolanski, N. On inhomogeneous minimization problems for the p(x)-Laplacian, in preparation.

[27] Lewis, J., \& Nystrom, K. Regularity of Lipschitz free boundaries in two phase problems for the p-Laplace operator, Adv. Math. 225 (2010), 2565-2597.

[28] Lyaghfouri, A. A minimum problem with free boundary for the $p(x)$-Laplace operator, Adv. Nonlinear Stud. 11 (2011), 25-61.

[29] Martínez, S., \& Wolanski, N. A minimum problem with free boundary in Orlicz spaces, Adv. Math. 218 (6) (2008), 1914-1971.

[30] Moreira, D., \& Wang L. Hausdorff measure estimates and Lipschitz regularity in inhomogeneous nonlinear free boundary problems, Arch. Ration. Mech. Anal. 213 (2014), 527-559.

[31] Omata, S. A free boundary problem for a quasilinear elliptic equation. I. Rectifiability of the free boundary, Differential Integral Equations 6 (6) (1993), 1299-1312.

[32] Omata, S., \& Yamaura, Y. A free boundary problem for nonlinear elliptic equations, Proc. Japan Acad. Ser. A Math. Sci. 66 (1990), 281-286.

[33] Ruzicka, M. Electrorheological Fluids: Modeling and Mathematical Theory, Springer-Verlag, Berlin, 2000.

[34] Wang, P. Y. Regularity of free boundaries of two-phase problems for fully nonlinear elliptic equations of second order. I. Lipschitz free boundaries are $C^{1, \alpha}$, Comm. Pure Appl. Math. 53 (7) (2000), 799-810.

[35] Weiss, G. S. Partial regularity for a minimum problem with free boundary, J. Geom. Anal. 9 (2) (1999), $317-326$.

[36] Wolanski, N. Local bounds, Harnack inequality and Hölder continuity for divergence type elliptic equations with non-standard growth, Rev. Un. Mat. Argentina 56 (1) (2015), 73-105.

[37] Zeldovich, Ya. B., \& Frank-Kamenetski, D. A. The theory of thermal propagation of flames, Zh. Fiz. Khim. 12 (1938), 100-105 (in Russian); English translation in "Collected Works of Ya. B. Zeldovich", vol. 1, Princeton Univ. Press, 1992.

imas - Conicet and Departamento de Matemática, Facultad de Ciencias Exactas y Naturales, Universidad de Buenos Aires, (1428) Buenos Aires, Argentina.

E-mail address, Claudia Lederman: clederma@dm.uba.ar

E-mail address, Noemi Wolanski: wolanski@dm.uba.ar 